\title{
HORMAD1 promotes docetaxel resistance in triple negative breast cancer by enhancing DNA damage tolerance
}

\author{
BEIGE ZONG ${ }^{1,2}$, LU SUN $^{1}$, YANG PENG ${ }^{1,2}$, YIHUA WANG ${ }^{1}$, YU YU $^{3}$, JINWEI LEI $^{1,2}$, \\ YINGZI ZHANG ${ }^{1,2}$, SHIPENG GUO ${ }^{2}, \mathrm{KANG} \mathrm{LI}^{1,2}$ and SHENGCHUN LIU ${ }^{1}$
}

\author{
${ }^{1}$ Department of Endocrine and Breast Surgery, The First Affiliated Hospital of Chongqing Medical University, \\ Chongqing 400042; ${ }^{2}$ Chongqing City Key Lab of Translational Medical Research in Cognitive Development and Disorders, \\ Children's Hospital of Chongqing Medical University, Chongqing 400016; \\ ${ }^{3}$ Department of Pathology, Chongqing Medical University, Chongqing 400015, P.R. China
}

Received December 10, 2020; Accepted April 28, 2021

DOI: $10.3892 /$ or.2021.8089

\begin{abstract}
HORMA domain-containing protein 1(HORMAD1), is normally expressed only in the germline, but is frequently re-activated in human triple-negative breast cancer (TNBC); however, its function in TNBC is largely unknown. In the present study, the expression and biological significance of HORMAD1 in human TNBC was evaluated. Bioinformatics analysis and reverse transcription-quantitative PCR were used to evaluate HORMAD1 expression in datasets and cell lines. HORMAD1 protein expression was detected in TNBC samples using immunohistochemical assays, and the effect of HORMAD1 on cell proliferation was determined using Cell Counting Kit-8, plate colony formation and standard growth curve assays. Cell cycle, reactive oxygen species (ROS) and apoptosis analyses were conducted using flow cytometry. The activity of caspases was measured using caspase activity assay kit. The levels of key apoptosis regulators and autophagy markers were detected by western blot analysis. TNBC cell survival and apoptosis were not influenced by small interfering RNA targeting HORMAD1 alone; however, HORMAD1 knockdown enhanced autophagy and docetaxel (Doc)-induced apoptosis, compared with the control group. Furthermore, higher ROS levels and caspase-3, -8 and -9 activity were detected in MDA-MB-436 TNBC cells with HORMAD1 knockdown upon exposure to Doc. The levels of the induced DNA damage marker $\gamma \mathrm{H} 2 \mathrm{AX}$ were also higher, while those of the DNA repair protein RAD51 were lower in TNBC cells with HORMAD1 knockdown compared with the controls. Furthermore, the expression of the autophagy marker P62 was enhanced in MDA-MB-231 cells in response to
\end{abstract}

Correspondence to: Dr Shengchun Liu, Department of Endocrine and Breast Surgery, The First Affiliated Hospital of Chongqing Medical University, Yuzhong, Yujiagang Youyi Road 1, Chongqing 400042, P.R. China

E-mail: liushengchun1968@163.com

Key words: triple-negative breast cancer, HORMA domaincontaining protein 1 , apoptosis, DNA damage, autophagy
HORMAD1 overexpression. Notably, Doc-induced apoptosis was similarly increased by both HORMAD1 overexpression and treatment with the autophagy inhibitor, 3-methyladenine (3MA); however, the Doc-induced increase in autophagy was not inhibited by 3MA. The present data indicated that HORMAD1 was involved in autophagy and that the inhibition of autophagy can partially enhance the induction of apoptosis by Doc. The role of HORMAD1 in the DNA damage tolerance of tumor cells may be the main reason for Doc resistance; hence, HORMAD1 could be an important therapeutic target in TNBC.

\section{Introduction}

Breast cancer has overtaken lung cancer as the most common type of cancer in women, with an estimated 2.3 million new cases in 2020, according to the latest cancer statistics report (1). Triple-negative breast cancer (TNBC) is a breast cancer subtype. Patients with TNBC exhibit a shorter median time to relapse and death, due to the lack of effective treatment targets. This lack of specific therapies is the leading cause of recurrence and mortality in patients with TNBC, alongside treatment resistance (2). In addition to surgical treatment, chemotherapy remains the main systematic treatment for breast cancer (3). Although several large randomized controlled trials have been conducted to explore novel treatment options for TNBC, such as programmed death ligand 1, a biomarker that has been included in the National Comprehensive Cancer Network (NCCN) guidelines (4), the results have been unsatisfactory. To date, taxanes remain the primary chemotherapy option for patients with TNBC in the clinic, as current international guidelines support the use of a single taxane and/or anthracyclines as first-line treatment $(5,6)$. Chemotherapy resistance and the lack of TNBC-targeted therapies lead to poor patient prognosis (7).

Multiple studies have demonstrated that the pathologic complete response ( $\mathrm{pCR}$ ) rate of TNBC to neoadjuvant chemotherapy (NAC) is superior to that of non-TNBC subtypes $(8,9)$ by up to $37.8 \%(10)$. NAC can not only lead to a reduction in the stage and shrinking of the breast tumor but also detect the sensitivity of the tumor to chemotherapy regimens. In 
early-stage breast cancer, the association between pCR rate and long-term survival is most obvious in patients with TNBC (4). The search for chemotherapy-sensitive markers and ways to overcome tumor resistance in TNBC are current research foci.

Cancer testis antigens (CTAs) are genes named after their expression patterns, since they are usually expressed only in the testes, where they play important roles in homologous chromosome recombination during meiosis, but also exhibit an abnormally high expression in a variety of malignant tumors (11). HORMA domain-containing protein 1 (HORMAD1; also referred to as CT46) is a CTA normally expressed in the testes, which has also been revealed to be expressed in female gametes and to have important physiological functions (12), with abnormally high levels in TNBC $(13,14)$. It has been indicated that HORMAD1 plays an important carcinogenic role in basal-like breast cancer (BLBC) (14) and it may induce a spontaneous antibody response in patients with cancer, due to its immunogenic properties (13). Meanwhile, several basic research studies have indicated that HORMAD1 can reduce or enhance the susceptibility of different tumors to docetaxel (Doc) and poly-ADP-ribose polymerase I inhibitor (PARPi) in different types of cancer (15-17). Therefore, in the present study, the association between the aberrant expression of HORMAD1 in tumors and the clinicopathological characteristics of patients was explored, and certain abnormal biological behaviors caused by the aberrantly high expression of HORMAD1 in tumor cells and its influence on the tolerance to common chemotherapy drugs were studied through a series of functional experiments, with the aim of providing evidence to support drug treatment choice for patients with TNBC and explore a new therapeutic target for TNBC.

\section{Materials and methods}

Bioinformatic methods and data analysis. Data derived from 1,208 breast cancer samples were downloaded from The Cancer Genome Atlas (TCGA) database (https://cancergenome.nih.gov). A detailed analysis of HORMAD1 expression was conducted as previously described (18). Gene expression data in breast cancer cell lines were obtained from the Broad Institute Cell Cancer Line Encyclopedia (CCLE; https://portals. broadinstitute.org/ccle).

TNBC samples. This study was a retrospective analysis. A total of 640 TNBC samples were obtained at the time of diagnosis at The First Affiliated Hospital of Chongqing Medical University between November 2013 and August 2018. Data from patients (20-76 years old) with TNBC with complete treatment records, who had undergone NAC combined with surgical treatment and subsequent chemotherapy, were selected. The exclusion criteria for all participants were as follows: i) Patients who achieved pCR after NAC; and ii) patients with previous cancer, concomitant cancer or bilateral breast cancer. Written informed consent was obtained from all patients and the study was approved (approval no. 2020-279) by the Ethics Committee of the First Affiliated Hospital of Chongqing Medical University (Chongqing, China). Tumor size was measured by B-mode ultrasound image or X-ray mammography. Response to chemotherapy was assessed according to RECIST 1.1 (19), to determine whether the patient had achieved pCR. All postoperative chemotherapy cycles were completed, and imaging re-examination was conducted regularly.

Immunohistochemical quantification of TNBC tissue samples. HORMAD1 expression levels and tumor infiltrating lymphocyte (TIL) numbers in TNBC samples were investigated using 4- $\mu \mathrm{m}$ thick tissue sections cut from paraffin wax-embedded specimens preserved by the Department of Pathology (First Affiliated Hospital of Chongqing Medical University). According to standard immunohistochemical operating procedures, sections were boiled at $100^{\circ} \mathrm{C}$ for $3 \mathrm{~min}$ for antigen retrieval in $0.01 \mathrm{M}$ citric acid buffer $(\mathrm{pH}$ 6.0) and quenched for endogenous peroxidase activity with $0.3 \% \mathrm{H}_{2} \mathrm{O}_{2}$ solution for $15 \mathrm{~min}$. The samples were then blocked for non-specific binding with $10 \%$ normal goat serum at room temperature for 15 min and incubated with specific rabbit primary antibody against HORMAD1 (1:500; cat. no. HPA037850; Merck KGaA) overnight at $4^{\circ} \mathrm{C}$. Subsequently, sections were treated with goat anti-rabbit secondary antibody (1:200, cat. no. PV-9000; Beijing Zhongshan Golden Bridge Biotechnology Co., Ltd.; OriGene Technologies, Inc.) for $30 \mathrm{~min}$ at room temperature. Following staining with diaminobenzidine (cat. no. ZLI-9018; Beijing Zhongshan Golden Bridge Biotechnology Co., Ltd.; OriGene Technologies, Inc.) at room temperature for 10-60 sec, representative images were captured using a Nikon Eclipse 80i light microscope (x200 magnification; Nikon Corporation). Each section was semi-quantified by a professional pathologist using a relative scale (13).

Cell lines. The MCF7, T47D,ZR75-1, SK-BR-3, MDA-MB-231, MDA-MB-436, BT549 and MDA-MB-468 human breast cancer cell lines were purchased from the American Type Culture Collection and cultured in DMEM (Thermo Fisher Scientific, Inc.) containing $10 \%$ fetal calf serum (Thermo Fisher Scientific, Inc.), $4.5 \mathrm{~g} / 1 \mathrm{D}$-glucose, L-glutamine and $110 \mathrm{mg} / \mathrm{l}$ sodium pyruvate in a humidified incubator with $5 \% \mathrm{CO}_{2}$ at $37^{\circ} \mathrm{C}$. The medium was refreshed every $2-3$ days. Subsequently, transfection experiments were performed.

HORMAD1 knockdown and overexpression. Cells were plated in six-well plates and transfected with specific small interfering RNAs (siRNAs) using LipoFiter ${ }^{\mathrm{TM}}$ Liposomal Transfection reagent (Hanbio Biotechnology Co., Ltd.), according to the manufacturer's instructions. Specific siRNA sequences were screened out from multiple candidate siRNA molecules and the sequences of the siRNAs (100 pM) used were as follows: siRNA-negative control (NC) sense, 5'-UUCUCCGAACGU GUCACGUTT-3' and antisense, 5'-ACGUGACACGUU CGGAGAATT-3'; HORMAD1-siRNA1 (si-HORMAD1\#1 sense, 5'-CCAUGAGUGCACUGGUAUUTT-3' and antisense, 5'-AAUACCAGUGCACUCAUGGTT-3') equal to si-HORMAD1 (si-HORMAD1\#2 sense, 5'-GCAUUCUCC UCAUUCGCAATT-3' and antisense, 5'-UUGCGAAUGAGG AGAGUGCTT-3'). All siRNA oligomers were purchased from Shanghai GenePharma Co., Ltd. A lentiviral vector (hU6-MCS-Ubiquitin-EGFP-IRES-puromycin; 1x10 ${ }^{8} \mathrm{TU} / \mathrm{ml}$; $\mathrm{MOI}=10$ ) including the same siRNA sequence was purchased from GeneChem, Inc., as certain experiments required that the knockout efficiency be maintained over a long period of 
time. Experiments were performed following transfection at $37^{\circ} \mathrm{C}$ for $72-96 \mathrm{~h}$. HORMAD1-overexpressing plasmid $(1 \mu \mathrm{g} / \mathrm{ml})$ was constructed at Chongqing City Key Lab of Translational Medical Research in Cognitive Development and Disorders (Children's Hospital of Chongqing Medical University, Chongqing, China) using the following primers: Forward, 5'TAAGCTTGGTACCGAGCTCGGATCCGCCAC CATGGCCACTGCCCAGTTG-3' and reverse, 5'ACGGGC CCTCTAGACTCGAGTTATATATGTTCCTTTGGTTCA CTAAACTTTCTCCTTTTTGG-3'. The pcDNA3.1 plasmid $(1 \mu \mathrm{g} / \mathrm{ml})$ was kindly provided by Dr Shipeng Guo and used as the vector to clone HORMAD1. Experiments were performed following transfection at $37^{\circ} \mathrm{C}$ for $24-48 \mathrm{~h}$, as indicated.

RNA isolation and reverse transcription-quantitative $(R T-q) P C R$. Total RNA was extracted from cancer cells using a Simply P Total RNA Extraction kit (cat. no. BSC52S1; Hangzhou Bioer Technology Co., Ltd.) and reverse-transcribed using the PrimeScript RT reagent kit according to the manufacturer's instructions. Fluorescence RT-qPCR was performed using SYBR Premix Ex Taq ${ }^{\mathrm{TM}}$ II (Takara Bio, Inc.) in a 10- $\mu 1$ PCR mixture on a Bio-Rad CFX96 Real-Time PCR system (Bio-Rad Laboratories, Inc.), according to the manufacturer's instructions. HORMAD1 gene-specific primers (forward, 5'-GCCCAGTTGCAGAGGACTC-3' and reverse, 5'-TCTTGTTCCATAAGCGCATTCT-3') were designed (17) and purchased from Thermo Fisher Scientific, Inc. GAPDH (forward, 5'-CTCTGCTCCTCCTGTTCGAC-3' and reverse, 5'-GCGCCCAATACGACCAAATC-3') was used as an endogenous control for each reaction. The cycling conditions were $94^{\circ} \mathrm{C}$ for $2 \mathrm{~min}, 39$ cycles of $95^{\circ} \mathrm{C}$ for $30 \mathrm{sec}$, $58^{\circ} \mathrm{C}$ for $30 \mathrm{sec}$ and $72^{\circ} \mathrm{C}$ for $20 \mathrm{sec}$, and $72^{\circ} \mathrm{C}$ for $7 \mathrm{~min}$. Each reaction was repeated three times. HORMAD1 expression was reported as a mean $\mathrm{Cq}$ (20) value (average fold-change relative to GAPDH) using CFX Manager ${ }^{\mathrm{TM}}$ software (version 3.0; Bio-Rad Laboratories, Inc.).

Cell proliferation, colony formation and drug susceptibility assays. Cell proliferation and drug-mediated inhibition were detected using the Cell Counting Kit-8 (CCK-8; MedChemExpress) assay, according to the manufacturer's instructions. Cells were plated at a density of $2-8 \times 10^{4}$ cells/well in 96-well plates, with or without different concentrations of Doc (cat. no. HY-B0011; MedChemExpress), and cell viability was assessed after $24 \mathrm{~h}$. The absorbance of each well at a wavelength $450 \mathrm{~nm}$ was measured using a Synergy $\mathrm{H} 1$ microplate reader (BioTek Instruments, Inc.). Empty wells containing the same volume of medium served as the blank controls. Cells were treated with different concentrations of Doc for various periods of time (0.1-40 nm; 0-96 h). The $\mathrm{IC}_{50}$ of Doc was calculated according to a standard curve and the most appropriate drug concentration was selected for subsequent experiments.

For the colony formation assay, $5 \times 10^{2}$ cells were plated in each of the six wells of the culture plate and incubated at $37^{\circ} \mathrm{C}$ for 1-2 weeks. Cells were then fixed with $4 \%$ paraformaldehyde at room temperature for $20 \mathrm{~min}$ and stained with $0.1 \%$ crystal violet (Merck $\mathrm{KGaA}$ ) at room temperature for $10 \mathrm{~min}$, and the cell colonies ( $>50$ cells/colony) were counted and analyzed by ImageJ v1.8.0 (National Institutes of Health).
Treatment with 3-methyladenine (3MA). The autophagy inhibitor 3MA was purchased from MedChemExpress (cat. no. HY-19312). Following $6 \mathrm{~h}$ after transfection with si-HORMAD1 or negative control siRNA (si-NC), cells were incubated with $10 \mu \mathrm{M} 3-\mathrm{MA}$ with or without $40 \mathrm{nM}$ Doc at $37^{\circ} \mathrm{C}$ for $24 \mathrm{~h}$. Cell proliferation was evaluated as aforementioned.

Treatment with Earle's balanced salt solution (EBSS). EBSS (cat. no. H2040; Beijing Solarbio Science \& Technology Co., Ltd.) was used to simulate the autophagy phenotype induced by cell starvation. The two TNBC cell lines (MDA-MB-468 and BT549) were placed in a six-well plate according to their growth conditions, and the growth density was $80-90 \%$ on the second day. The complete medium was replaced with calciumand phosphorus-free EBSS, and the cells were collected for protein extraction after being treated for different time-points $(0,2$ and $4 \mathrm{~h})(21)$.

Flow cytometry. The cell cycle was analyzed by flow cytometry. Briefly, following transfection with si-NC or si-HORMAD1 for $48 \mathrm{~h}$, cells were exposed to Doc $(40 \mathrm{nM})$ at $37^{\circ} \mathrm{C}$ for $24 \mathrm{~h}$. Cells were then digested with trypsin and washed twice with ice-cold PBS, and 75\% alcohol was added to the cell suspension, followed by vortex mixing. Fixed cells were suspended in PI $(100 \mathrm{mg} / \mathrm{ml}$; excitation wavelength, $488 \mathrm{~nm}$; emission wavelength, $630 \mathrm{~nm})$ and RNase $(10 \mathrm{mg} / \mathrm{ml})$ and incubated at $37^{\circ} \mathrm{C}$ for $30 \mathrm{~min}$ in the dark. All samples were assessed using a FACSCanto system (BD Biosciences). Data were analyzed using Cell Quest software 5.1 (BD Biosciences).

Detection of apoptosis. Apoptosis was measured using an Annexin V-FITC/7-AAD kit (cat. nos. FXP014 and FXP145; 4A Biotech., Co., Ltd.). Cells were treated with $40 \mathrm{nM}$ Doc following transfection with si-NC or si-HORMAD1 for $48 \mathrm{~h}$ or lentiviral transfection for $96 \mathrm{~h}$. The cells were then collected and resuspended in medium, washed twice with cold PBS, and suspended in binding buffer at a cell density of $1 \times 10^{6}$ cells $/ \mathrm{ml}$. Cells were stained with $5 \mu \mathrm{l}$ Annexin V-FITC at room temperature for $5 \mathrm{~min}$ and $10 \mu \mathrm{lPI}(20 \mu \mathrm{g} / \mathrm{ml})$ at room temperature for $5 \mathrm{~min}$, according to the manufacturer's instructions. Samples were analyzed by flow cytometry (FACSCanto) following incubation in the dark at room temperature for $10 \mathrm{~min}$. The proportion of apoptotic cells was determined using FlowJo_V10 (FlowJo LLC), with 30,000 cells analyzed per well.

Detection of reactive oxygen species (ROS). Intracellular ROS levels in the form of cellular peroxides were assessed using a ROS Assay kit according to the manufacturer's instructions (cat. no. D6883; Merck KGaA), following treatment with or without Doc. MDA-MB-436 cells were treated with $40 \mathrm{nM}$ Doc for $24 \mathrm{~h}$, at $48 \mathrm{~h}$ after the siRNA transfection. Cells were collected, exposed to $10 \mu \mathrm{M} 2$ ',7'-dichlorofluorescein diacetate, and incubated at $37^{\circ} \mathrm{C}$ for $20 \mathrm{~min}$. They were then washed three times with cold PBS, and fluorescence intensity was analyzed by flow cytometry (FACSanto657338). ROS levels are expressed as the mean fluorescence intensity of 30,000 cells.

Evaluation of caspase activity. Cell caspase activity was measured using a Caspase-3/8/9 Activity Assay kit (cat. nos. C1115/C1152/C1157, respectively; Beyotime Institute of 
Biotechnology). Cells were treated with Doc $(40 \mathrm{nM}) 24 \mathrm{~h}$ after transfection with si-NC or si-HORMAD1, and total cells were collected according to the manufacturer's instructions. Next, $100 \mu \mathrm{l}$ cell lysate and test reagent per well was incubated in 96 -well plates at $37^{\circ} \mathrm{C}$ for $2 \mathrm{~h}$. Caspase activity was determined using an enzyme standard instrument at $405 \mathrm{~nm}$ and transformed using a standard conversion curve.

Western blot analysis. Cells were washed once with cold PBS before harvesting. Protein lysates were prepared from MDA-MB-436, MDA-MB-468, BT549 and MDA-MB-231 cells using a total protein extraction kit (cat. no. KGP2100; Nanjing KeyGen Biotech, Co., Ltd.) according to the manufacturer's instructions, and protein concentration was assessed using a Pierce ${ }^{\mathrm{TM}}$ BCA Protein Assay kit (Thermo Fisher Scientific, Inc.), followed by standard western blot analysis, as outlined below. Protein samples (30 $\mu \mathrm{g} / \mathrm{lane})$ were separated by $10-12 \%$ SDS-PAGE and transferred to $0.2-\mu \mathrm{m}$ PVDF membranes (EMD Millipore). Following blocking with 5\% skim milk for $1 \mathrm{~h}$ at room temperature, the membranes were incubated with specific primary antibodies at $4^{\circ} \mathrm{C}$ overnight. Next, following washing 3 times with Tris-buffered saline containing $0.1 \%$ Tween-20, the membranes were incubated with horseradish peroxidase-conjugated secondary antibodies for $1 \mathrm{~h}$ at room temperature. Proteins were visualized by chemiluminescence, using enhanced chemiluminescent substrate (cat. no. P10300; New Cell \& Molecular Biotech Co., Ltd.). Immunoreactive bands were examined using the ChemiDoc Imaging System (Bio-Rad Laboratories, Inc.). The primary antibodies used included HORMAD1 (1:1,000; cat. no. HPA037850; Merck KGaA), Bax (1:2,000; cat. no. 50599-2-Ig; ProteinTech Group, Inc.), Bcl-2 (1:500; cat. no. 60178-1-lg; ProteinTech Group, Inc.); P62 (1:1,000; cat no. 66184-1-lg; ProteinTech Group, Inc.), Beclin 1 (1:1,000; cat. no. 11306-1-AP; ProteinTech Group, Inc.), light chain 3 (LC3; 1:1,000; cat. no. 12741S; Cell Signaling Technology, Inc.), RAD51 (1:1,000; cat. no. 14961-1-AP; ProteinTech Group, Inc.), phospho-histone $\mathrm{H} 2 \mathrm{AX}-\mathrm{S} 139$ also named $\gamma \mathrm{H} 2 \mathrm{AX}(1: 1,000$; cat. no. AP0099; ABclonal Biotech Co., Ltd.), cleaved caspase-3 (1:1,000; cat. no. 25546-1-AP; ProteinTech Group, Inc.), GAPDH (1:5,000; cat. no. 10494-1-AP; ProteinTech Group, Inc.) and $\beta$-actin (1:5,000; cat. no. 20536-1-AP; ProteinTech Group, Inc.). The secondary antibodies used were horseradish peroxidase-conjugated goat anti-rabbit antibodies (1:3,000; cat. no. 7074S; Cell Signaling Technology, Inc.). GAPDH or $\beta$-actin was used as an internal control for each blot. ImageJ software v1.8.0 was used to quantify western blot analysis, and GraphPad Prism 7 software (GraphPad Software, Inc.) was used for plotting and statistical analysis.

Hoechst staining. Hoechst stain was measured using TUNEL Apoptosis Assay Kit (cat. no. T2190; Beijing Solarbio Science \& Technology Co., Ltd.). Cells were transfected with si-NC or si-HORMAD1 for 24-48 $\mathrm{h}$ and then plated at a density of $2-3 \times 10^{5}$ cells/well in $24-w e l l$ plates with a sterile glass slipper at the bottom for 12-24 h. They were then treated with or without Doc at $37^{\circ} \mathrm{C}$ for $24 \mathrm{~h}$. Subsequently, the Hoechst staining was carried out according to the manufacturer's instructions. Briefly, TNBC cells were fixed with a $4 \%$ paraformaldehyde fixation solution at room temperature for $20 \mathrm{~min}$, and then rinsed three times with $0.01 \mathrm{~mol} / \mathrm{l} \mathrm{PBS}$ for $5 \mathrm{~min}$. Cells were then stained with $1 \mathrm{X}$ Hoechst working solution at room temperature for $15 \mathrm{~min}$ in the dark, followed by washing three times with PBS, for 5 min each time. Next, anti-fluorescence quenching agents were used to fix the cell slide onto the cover glass and observed under a fluorescence microscope (magnification, x200). The dye was a DNA-specific fluorescent probe, and the nucleus exhibited bright blue fluorescence. The number of nuclear fragmentations in three random fields were observed and counted, and the results were statistically analyzed.

Statistical analysis. Survival analysis was carried out using R2 (http://r2.amc.nl), a genomics analysis and visualization platform. All experiments were performed independently at least three times. Statistical analyses were performed using GraphPad Prism 7.0 (GraphPad Software Inc.). Metrological data are presented as the mean \pm SD. An unpaired Student's t-test was used for two individual comparisons and the mean of multiple groups was compared by one-way analysis of variance (ANOVA). Multiple comparisons were performed using Bonferroni correction following ANOVA. The categorical variables are presented as numbers and percentages and were compared using $\chi^{2}$ and Fisher's tests. $\mathrm{P}<0.05$ was considered to indicate a statistically significant difference.

\section{Results}

Expression level of HORMADI in TNBC. To evaluate HORMAD1 expression in breast cancer, bioinformatics analysis was first conducted. As shown in Fig. 1, compared with paired peritumoral controls, there was no significant difference in HORMAD1 expression among the Luminal A $(n=40$, $\mathrm{P}>0.05)$, Luminal $\mathrm{B}(\mathrm{n}=30, \mathrm{P}>0.05)$ or human epidermal growth factor receptor 2 overexpression $(n=17, P>0.05)$ breast cancer subtypes, while HORMAD1 expression was significantly higher in TNBC tumors compared with adjacent normal tissue ( $\mathrm{n}=20, \mathrm{P}<0.001$; Fig. 1A). These data demonstrated that HORMAD1 was abnormally, and almost exclusively, expressed at higher levels in TNBC tumors; however, no association was detected between HORMAD1 levels and the prognosis of patients with breast cancer (Fig. 1B).

Next, bioinformatics analysis was performed to evaluate HORMAD1 expression in breast cancer cell lines from the CCLE. The results demonstrated similar trends to that of tumor tissue analyses, in that HORMAD1 was almost exclusively highly expressed in TNBC or BLBC cell lines, including BT549 and MDA-MB-436 (Fig. 1C).

Next, HORMAD1 mRNA and protein expression were tested in various breast cancer cell lines commonly used in the laboratory. A high HORMAD1 expression was only detected in MDA-MB-468 cells, with no HORMAD1 expression revealed in non-TNBC cell lines ( $\mathrm{P}<0.001$; Figs. 1D and S1A), which was consistent with a previous study (14).

Current chemotherapy regimens for TNBC include both Doc and anthracyclines $(14,15)$. Therefore, TNBC cases treated with NAC were selected for comparative expression analysis, according to specific inclusion and exclusion criteria as aforementioned. Finally, 154 TNBC cases were enrolled, among which the pCR rate was $24.0 \%$ (37/154). 


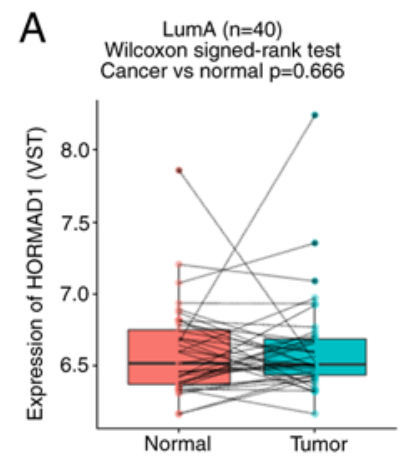

B Strata $->6.51(121)-<6.51(130)$

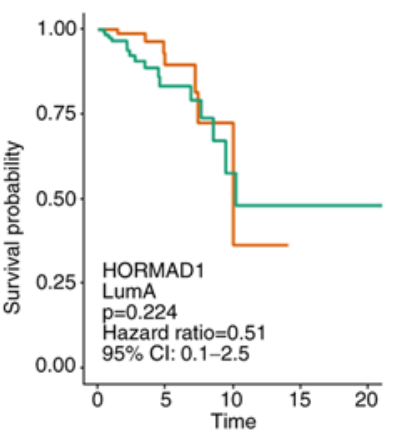

C

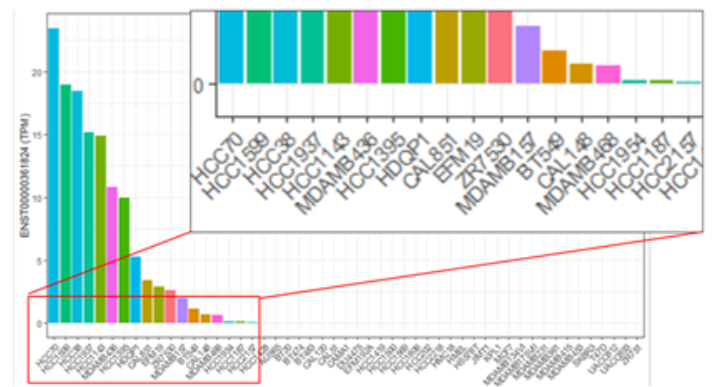

Strata $\rightarrow>6.43(111)-<6.43(71)$

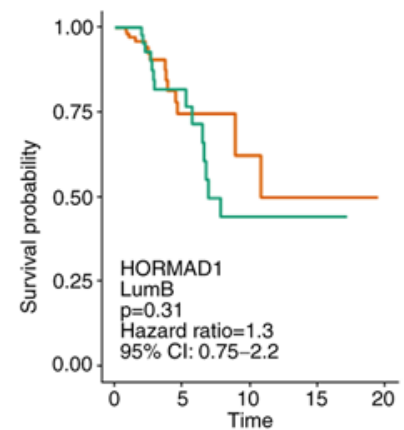

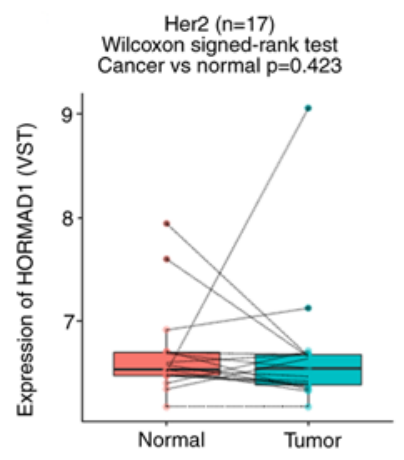
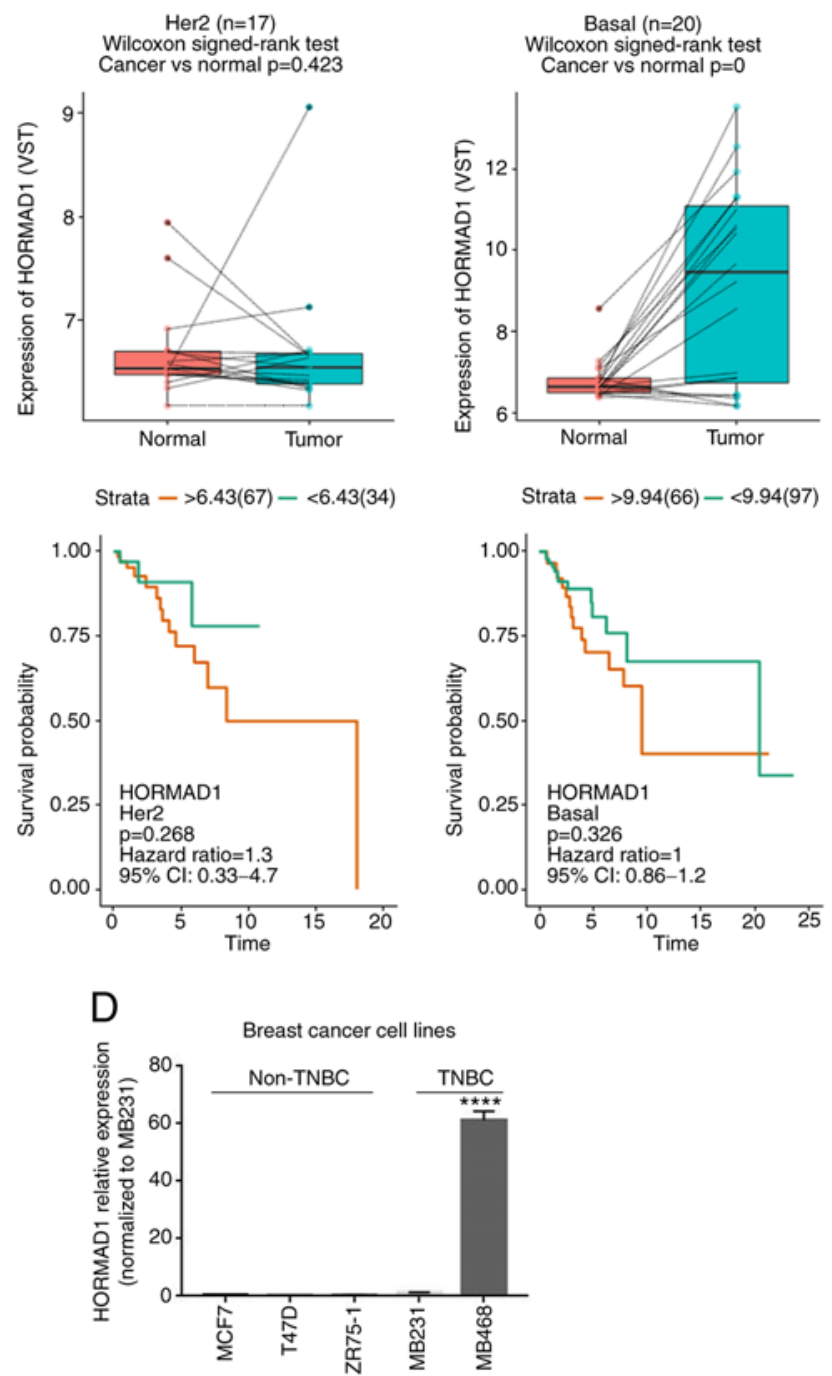

$\mathrm{E}$
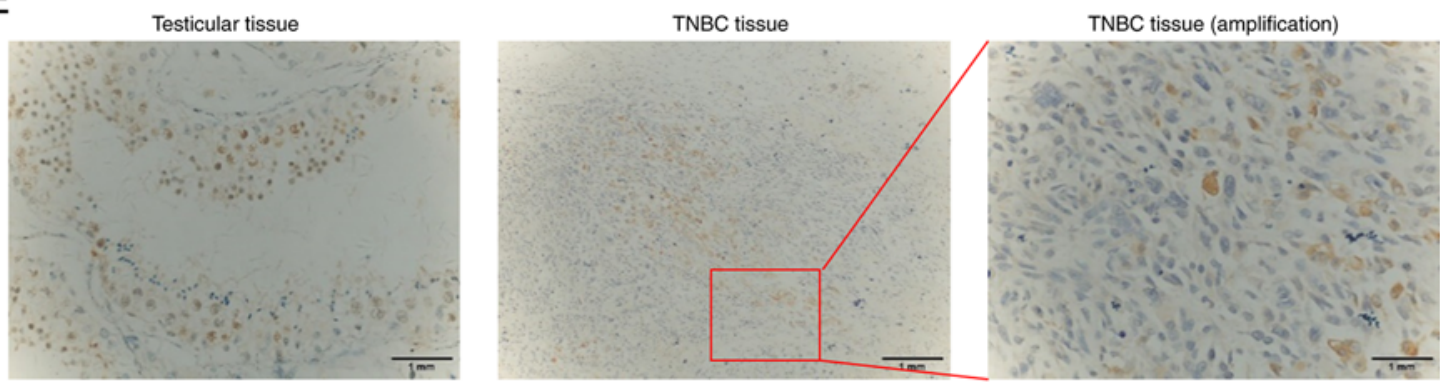

Figure 1. HORMAD1 expression in TCGA database and clinical samples. (A) Paired boxplot of HORMAD1 expression in 107 samples of different breast cancer subtypes. There was a significant difference in TNBC samples compared with the other three subtypes. (B) Kaplan-Meier analysis of overall survival was performed for different breast cancer subtypes using the R2 database, and no significant differences were observed. (C) HORMAD1 expression in the majority of breast cancer cell lines in the CCLE (https://portals.broadinstitute.org/ccle). The enlarged images shown in the red box are all TNBC cell lines. (D) HORMAD1 gene expression in certain breast cancer cell lines was determined by reverse transcription-quantitative PCR using HORMAD1 primers. A high HORMAD1 expression was only detected in MDA-MB-468 cells. (E) HORMAD1 protein expression in tissues determined by immunohistochemistry. The HORMAD1 positive control (normal testis) is presented on the left (magnification, x100) and the expression of HORMAD1 in one TNBC sample is presented in the middle (magnification, $\mathrm{x} 100$ ) and right (magnification, $\mathrm{x} 400$ ) images. ${ }^{* * * *} \mathrm{P}<0.001$. HORMAD1, HORMA domain-containing protein 1 ; TCGA, The Cancer Genome Atlas; CCLE, Cell Cancer Line Encyclopedia; TNBC, triple-negative breast cancer.

To further explore the expression of HORMAD1 in patients with TNBC with residual disease, paraffin-embedded tumor biopsy specimens were sectioned from 109 cases ( 8 cases were excluded due to the lack of tissue availability) without pCR for hematoxylin and eosin (preserved at the Department of Pathology, First Affiliated Hospital of Chongqing Medical
University), and immunohistochemical staining; TIL scores were determined concurrently. High levels of HORMAD1 expression were specifically detected in the spermatogenic positive control (Fig. 1E, left), and HORMAD1 protein expression was also detected in some TNBC tissue samples (Fig. 1E, right). Furthermore, statistical analysis demonstrated 
Table I. Associations between HORMAD1 expression and clinicopathologic features of 109 TNBC cases.

\begin{tabular}{|c|c|c|c|}
\hline \multirow[b]{2}{*}{ Characteristics } & \multicolumn{2}{|c|}{ HORMAD1 expression } & \multirow[b]{2}{*}{ P-value } \\
\hline & Negative $n=83(\%)$ & Positive $\mathrm{n}=26(\%)$ & \\
\hline Age at diagnosis (years) & & & 0.216 \\
\hline$\leq 50$ & $53(63.9)$ & $20(76.9)$ & \\
\hline$>50$ & $30(36.1)$ & $6(23.1)$ & \\
\hline Menopausal status & & & 0.197 \\
\hline Pre/peri & $49(59.0)$ & $19(73.1)$ & \\
\hline Post & $34(41.0)$ & $7(26.9)$ & \\
\hline Histologic subtype & & & 0.491 \\
\hline IDC & $74(87.1)$ & $25(96.2)$ & \\
\hline No IDC & $9(10.8)$ & $1(3.8)$ & \\
\hline Post-NAC tumor size $(\mathrm{cm})$ & & & 0.856 \\
\hline$\leq 2.0$ & $40(48.2)$ & $12(46.2)$ & \\
\hline$>2.0$ & $43(51.8)$ & $14(53.8)$ & \\
\hline Post-NAC lymph node involvement & & & 0.251 \\
\hline Yes & $31(37.3)$ & $13(51.9)$ & \\
\hline No & $52(62.7)$ & $13(48.1)$ & \\
\hline Post-Ki67 expression & & & 0.500 \\
\hline$\leq 14 \%$ & $26(31.3)$ & $10(38.5)$ & \\
\hline$>14 \%$ & $57(68.7)$ & $16(61.5)$ & \\
\hline Ki67 status & & & 0.844 \\
\hline Decrease & $59(71.1)$ & $19(73.1)$ & \\
\hline No decrease & $24(28.9)$ & $7(26.9)$ & \\
\hline Curative effect of NAC & & & 0.903 \\
\hline No & $34(41.0)$ & $11(42.3)$ & \\
\hline Yes & $49(59.0)$ & $15(57.7)$ & \\
\hline RD TILs level & & & 0.341 \\
\hline$<30 \%$ & $44(53.0)$ & $11(42.3)$ & \\
\hline$\geq 30$ & $39(47.0)$ & $15(57.7)$ & \\
\hline
\end{tabular}

HORMAD1, HORMA domain containing protein 1; TNBC, triple-negative breast cancer; TILs, tumor infiltrating lymphocytes; IDC, invasive ductal carcinoma; NAC, neoadjuvant chemotherapy; RD, residual disease.

that HORMAD1 was expressed in $23.9 \%(26 / 109)$ of patients with TNBC who had residual disease (Table I), similar to the $29.7 \%$ expression rate reported in samples from patients with untreated TNBC (22). No associations were found between HORMAD1 expression and patient clinicopathological characteristics (Table I).

TILs are a manifestation of host immune system recognition and defense against malignant cells (23). Several studies have reported that HORMAD1 was a potential neoantigen $(24,25)$; however, Pearson correlation coefficient testing demonstrated no correlation between HORMAD1 and the number of TILs ( $\mathrm{R}=0.09, \mathrm{P}=0.345)$ (data not shown). In addition, survival analysis indicated that HORMAD1 expression does not represent a prognostic factor for patients with TNBC (data not shown). Notably, it was revealed that the TIL number was an independent prognostic factor for both progression-free survival and overall survival in patients with primary TNBC, particularly those without a decreased Ki67 index following
NAC (data not shown); however, to the best of our knowledge, the role of HORMAD1 in TNBC biology remains unknown. High HORMAD1 expression cell lines were selected for use in follow-up studies, due to their high HORMAD1 expression levels (Fig. 1C).

Biological effect of HORMADI on the Doc sensitivity of TNBC cells. Due to the potential oncogenic role of HORMAD1 in breast carcinoma $(13,16)$, a series of experiments was conducted to understand the biological effects of HORMAD1 depletion. Specific siRNA targeting HORMAD1 (si-HORMAD1, equal to si-HORMAD1\#1) was used to reduce the expression of endogenous HORMAD1 $(\mathrm{P}<0.001$; Fig. 2A). To determine whether targeting HORMAD1 can decrease TNBC cell viability and potentially complement and improve the efficacy of Doc treatment, a series of experiments was carried out. MDA-MB-436 and MDA-MB-468 cell proliferation were not affected by HORMAD1 knockdown 

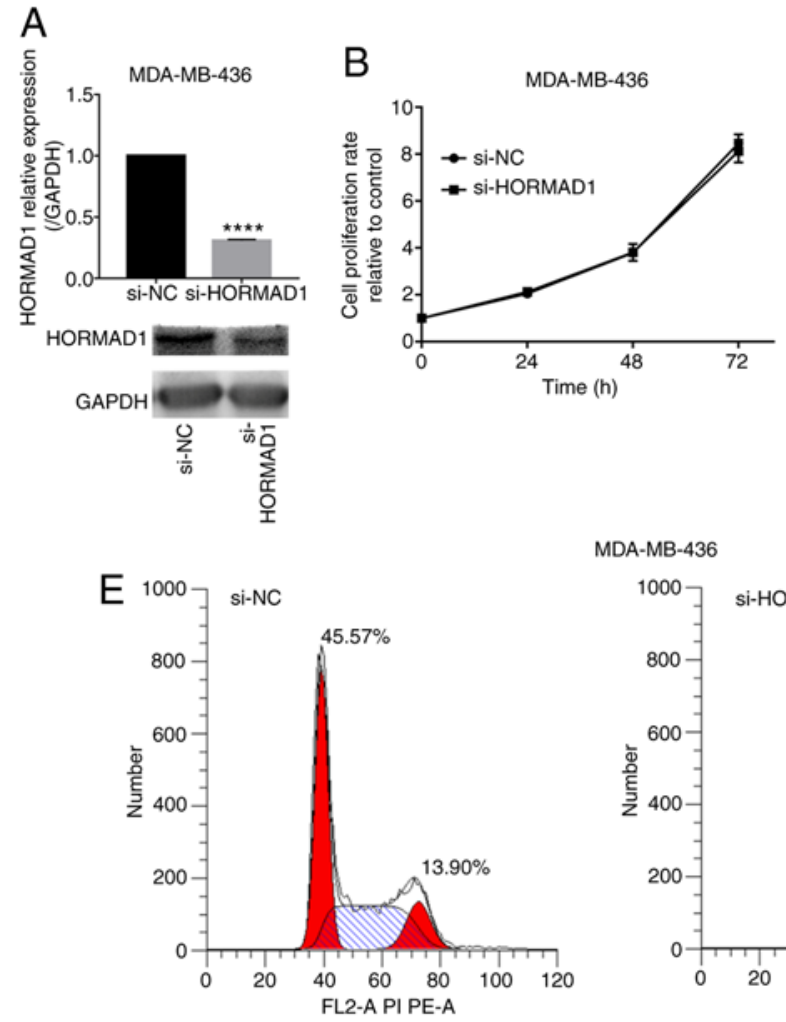

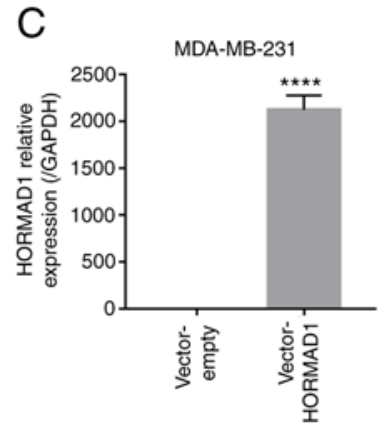

G
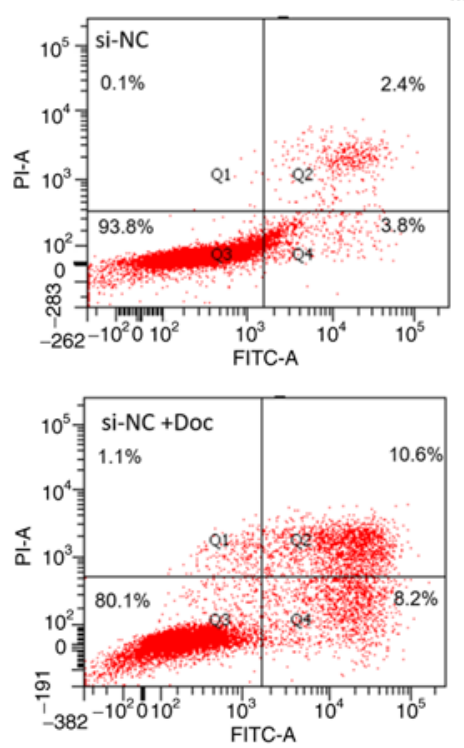

MDA-MB-436

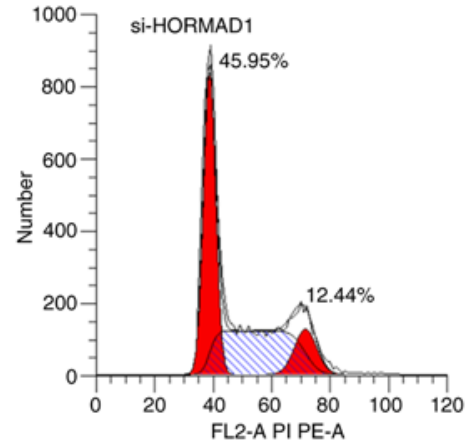

MDA-MB-436
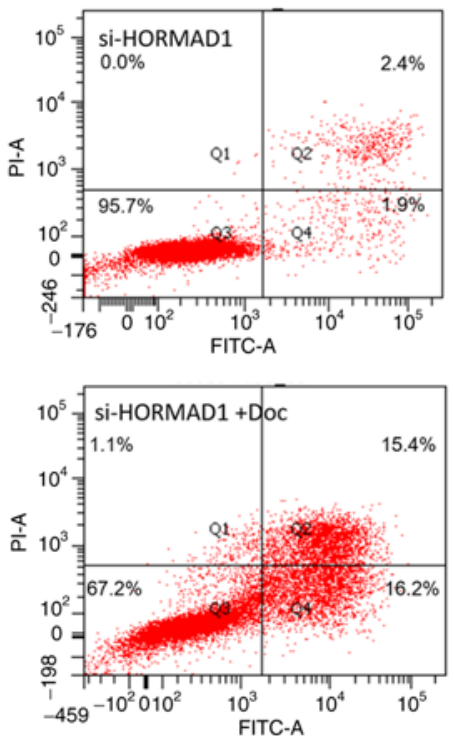

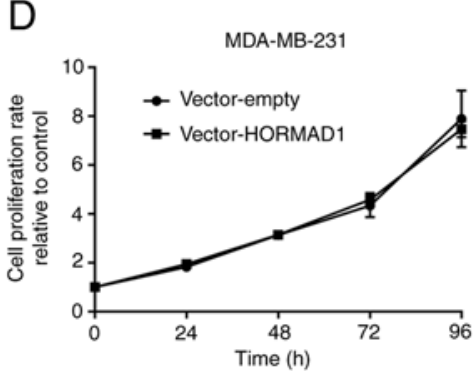

F

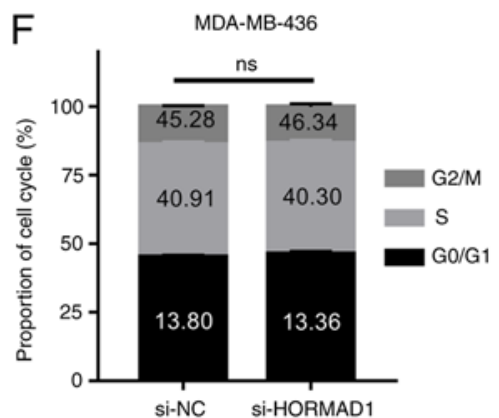

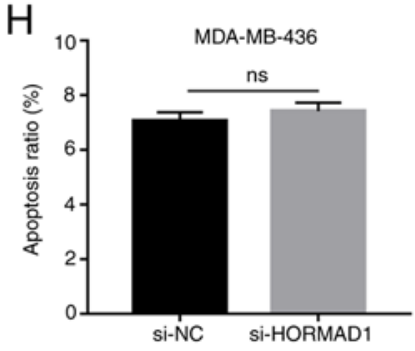

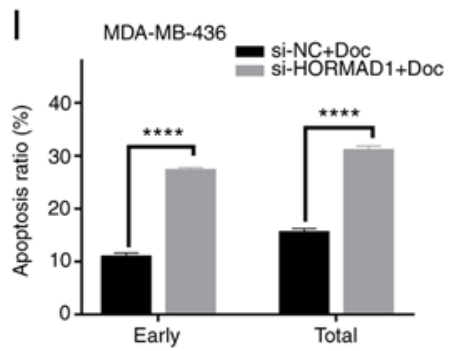

Figure 2. Biological effects of HORMAD1 in triple-negative breast cancer. (A) Specific si-HORMAD1 knockdown in MDA-MB-436 cells, as examined by RT-qPCR and western blot analysis. (B) The proliferation rate of MDA-MB-436 cells was not affected by HORMAD1 expression knockdown. (C) HORMAD1 overexpression in MDA-MB-231 cells using a specific overexpression plasmid by RT-qPCR. (D) The MDA-MB-231 cell proliferation rate was not affected by HORMAD1 overexpression. (E) Representative images of the MDA-MB-436 cell cycle with si-NC/HORMAD1. (F) Cell cycle data from flow cytometry presented as a histogram plot. There was no significant difference in the MDA-MB-436 cell cycle between the HORMAD1 knockdown and control groups. (G) Representative images of MDA-MB-436 cell apoptosis. (H) Apoptosis analysis through flow cytometry presented as a histogram. Compared with the control group, HORMAD1 knockdown did not influence MDA-MB-436 cell apoptosis; the histogram demonstrated the lack of a significant difference. (I) Compared with the controls, HORMAD1 knockdown led to a higher apoptotic rate in docetaxel-exposed cells (40 $\mathrm{nM})$ for $48 \mathrm{~h}$. Error bars represent the standard deviation and were derived from three independent experiments. ${ }^{* * * *} \mathrm{P}<0.001$. HORMAD1, HORMA domain-containing protein 1 ; si-HORMAD1, small interfering RNA-HORMAD1; RT-qPCR, reverse transcription-quantitative PCR; si-NC, siRNA negative control; ns, no significance.

alone, compared with si-NC (P>0.05; Figs. 2B and S1B). Consistent results were obtained using a colony formation assay (Fig. S1D). Flow cytometry indicated that a relatively high proportion of MDA-MB-436 cells was in the $G_{0} / G_{1}$ and $S$ phases compared with the $\mathrm{G}_{2} / \mathrm{M}$ phase, and that HORMAD1 knockdown did not affect the proportion of MDA-MB-436 cells in each phase (S-phase, $40.91 \pm 0.909$ vs. $40.30 \pm 1.15 \%$; $\mathrm{P}>0.05$; Fig. 2E and F). Consistent results were obtained using another 
breast cancer cell line expressing high HORMAD1 levels, MDA-MB-468 (S-phase, 10.67 \pm 0.142 vs. 10.34 $\pm 0.166 \%$; $\mathrm{P}>0.05$; Fig. S1C).

Similarly, HORMAD1 overexpression in MDA-MB-231 cells, using the pcDNA3.1 plasmid vector $(\mathrm{P}<0.001$; Fig. $2 \mathrm{C})$, did not affect MDA-MB-231 cell proliferation (Fig. 2D), which was consistent with the findings of a previous study that used SUM159 cells (16). Furthermore, there was no significant difference in total apoptosis between MDA-MB-436 cells with and without HORMAD1 knockdown $(7.06 \pm 0.185 \%$ vs $7.42 \pm 0.181 \%$, P>0.05; Fig. $2 \mathrm{G}$ and H). Collectively, compared with the siRNA-NC, siRNA-HORMAD1 had no effect on cell survival; however, similar to the findings of a study in epithelial ovarian cancer cells (15), exposure to Doc for $48 \mathrm{~h}$ resulted in a noticeable increase in MDA-MB-436 cell apoptosis, compared with negative controls (early, $11.07 \pm 0.35$ vs. $21.19 \pm 0.39 \%$, $\mathrm{P}<0.001$; total, $15.67 \pm 0.35$ vs. $27.4 \pm 0.21 \%, \mathrm{P}<0.001$; Fig. 2G and I). Finally, additional HORMAD1 silencing resulted in an apoptotic rate that was almost double compared with that caused by Doc treatment alone (Fig. 2I). These results indicated that HORMAD1 may exert biological effects on the sensitivity of MDA-MB-436 cells to chemotherapy drugs.

Effect of HORMADl on Doc-induced changes in TNBC cell apoptosis. To determine whether HORMAD1 expression influences Doc sensitivity, specific siRNAs were used to knock down HORMAD1 in MDA-MB-436 and BT549 cells, which were then treated with $40 \mathrm{nM}$ Doc (based on the $\mathrm{IC}_{50}$ value of MDA-MB-436; Fig. S2A). CCK-8 assays were used to investigate whether there was a difference in Doc sensitivity of cells with or without HORMAD1 knockdown, based on MDA-MB-436 cell proliferation rates. Compared with the si-NC group, the si-HORMAD1 group demonstrated lower proliferation rates following treatment with various concentrations of Doc for $24 \mathrm{~h}$, in both MDA-MB-436 (40 nM, 81.23 \pm 2.488 vs. $61.78 \pm 1.095 \%, \mathrm{P}<0.005$; Fig. $3 \mathrm{~A})$ and MDA-MB-468 (40 nM, 67.32 \pm 1.422 vs 36.89 $\pm 2.46, \mathrm{P}<0.001$; Fig. S2B) cells. Similar results were confirmed by growth curve analysis of MDA-MB-436 cells treated with or without lentiviral-HORMAD1 (RNAi-HORMAD1) at the same concentration as that of Doc $(96 \mathrm{~h}, 4.08 \pm 0.125$ vs. $2.85 \pm 0.070$, $\mathrm{P}<0.01$; Fig. 3C). The same result trends were observed in BT549 cells using two different specific siRNAs (Fig. 3B and D). These results demonstrated that the reduction of HORMAD1 expression could increase the drug sensitivity of TNBC cells positively expressing HORMAD1 to Doc.

Flow cytometry confirmed that there was a significant difference in the apoptotic rate between MDA-MB-436 cells with or without RNAi-HORMAD1 treatment exposed to Doc for $24 \mathrm{~h}$ (early apoptosis, $13.8 \pm 0.058$ vs. $19.43 \pm 0.567 \%$, $\mathrm{P}<0.005$; total apoptosis, $20 \pm 0.306$ vs. $25.7 \pm 0.651 \%, \mathrm{P}<0.01$; Fig. 3E and F). The same result trends were also observed in BT549 cells using specific siRNA (Fig. 3G).

Compared with Doc for $6 \mathrm{~h}$, Doc for $24 \mathrm{~h}$ produced obvious apoptotic signals. Moreover, cleaved caspase-3 levels were significantly increased in the si-HORMAD1 group compared with the NC group in MDA-MB-436 cells (Fig. 3H). Meanwhile, a similar phenomenon was observed in MDA-MB-468 cells (Fig. S2C). Analysis of common markers of apoptosis revealed that $\mathrm{Bcl}-2 / \mathrm{Bax}$ was significantly decreased in the si-HORMAD1+Doc group (Fig. 3I). These results indicated that HORMAD1 knockdown could promote the sensitivity of TNBC cells to Doc.

HORMADI depletion is associated with autophagy in certain $T N B C$ cells. Autophagy is closely associated with drug resistance (26). To explore whether HORMAD1 can affect TNBC drug sensitivity through autophagy, changes in autophagy markers in TNBC cells treated with si-NC or si-HORMAD1 were examined. Significant changes were observed in several typical autophagy markers in MDA-MB-436 and MDA-MB-468 cells with on HORMAD1 knockdown (Fig. 4A and B). The expression level of P62 was significantly decreased $(\mathrm{P}<0.05)$, while that of Beclin 1 was significantly increased $(\mathrm{P}<0.05)$, compared with the control group. Conversely, HORMAD1 overexpression in MDA-MB-231 cells, led to a significant increase in P62 compared with control cells $(\mathrm{P}<0.05$; Fig. 4C and D), while the addition of Doc was also accompanied by autophagy. It was hypothesized that HORMAD1 can inhibit autophagy in TNBC. Flow cytometry revealed that HORMAD1 overexpression in MDA-MB-231 cells had the same effect as the addition of the autophagy inhibitor 3MA in MDA-MB-436 cells (Fig. 4F and G), both of which increased Doc-induced apoptosis to a similar degree (MDA-MB-231, 1 vs. 1.27 $\pm 0.007, \mathrm{P}<0.001$; MDA-MB-436, 1 vs. $1.22 \pm 0.05, \mathrm{P}<0.05$; Fig. $4 \mathrm{E}$ and $\mathrm{H}$ ), indicating the inhibition of autophagy could increase drug sensitivity.

Cell viability assessment also indicated that Doc-induced cell damage was increased by $3 \mathrm{MA}$, while this effect was attenuated with increasing concentrations of Doc (20 nM, 61.17 \pm 1.1 .193 vs. $53.05 \pm 0.833 \%, \mathrm{P}<0.01 ; 40 \mathrm{nM}$, $60.1 \pm 0.819$ vs. $57.24 \pm 0.54 \%, \mathrm{P}<0.05$; Fig. $4 \mathrm{I})$. Drug sensitivity was further detected following HORMAD1 knockdown. However, in the HORMAD1-knockdown group, Doc-induced cell damage was not affected by the addition of 3MA (40 nM, $47.78 \pm 1.556$ vs. $47.35 \pm 1.496 \%$, P >0.05; Fig. $4 J$ ). These results indicated that the enhancement of autophagy induced by HORMAD1 knockdown was not responsible for its effects in sensitizing cells to Doc. Therefore, the present study aimed at exploring the reason why HORMAD1 plays a role in Doc resistance in tumors through its basic physiological functions.

However, not all TNBC cell lines with a high HORMAD1 expression exhibited this phenomenon, as for instance BT549 cells (Fig. S1E). In addition, compared with autophagy induced by EBSS-induced starvation (Fig. S1F), the autophagy associated with HORMAD1 knockdown may be more complex.

HORMAD1 depletion promotes Doc-induced DNA damage in TNBC cells. Next, the mechanism through which HORMAD1 influences TNBC cell resistance to Doc was explored. Since lung cancer cells expressing high levels of HORMAD1 can achieve resistance to piericidin A by reducing ROS-processing mechanisms (24), a ROS assay kit was used to detect differences in ROS levels in MDA-MB-436 cells treated with si-NC or si-HORMAD1 exposed to Doc. The intracellular ROS production induced by Doc was higher in cells with

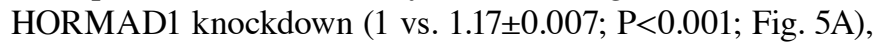
indicating that, under Doc-induced oxidative stress conditions, HORMAD1 may participate in the upregulation of antioxidant enzyme systems in TNBC cells to enhance anti-apoptosis, 


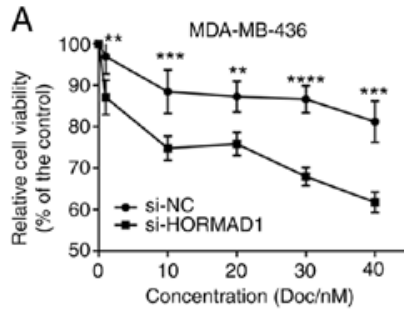

C

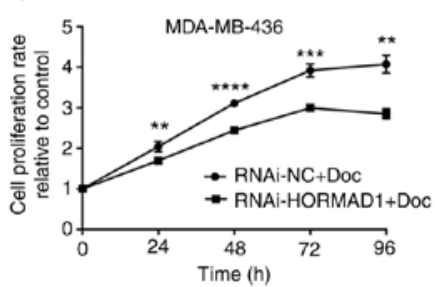

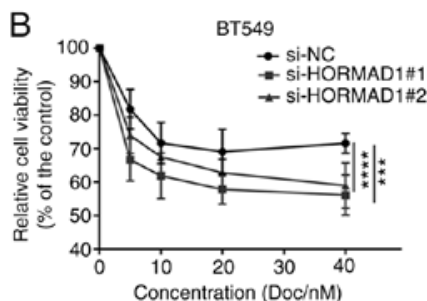

D

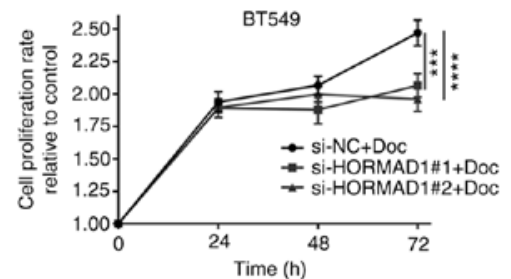

E

MDA-MB-436
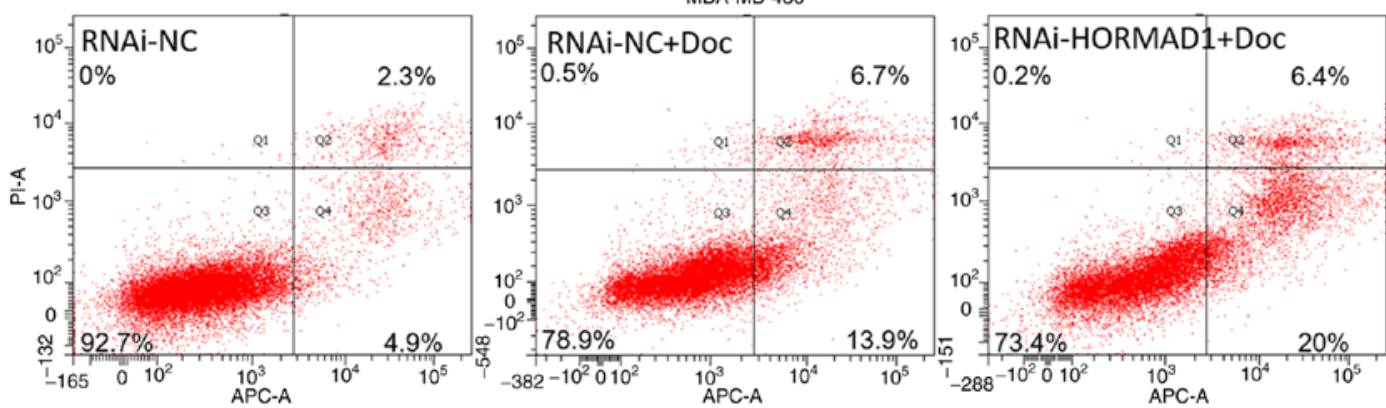

F

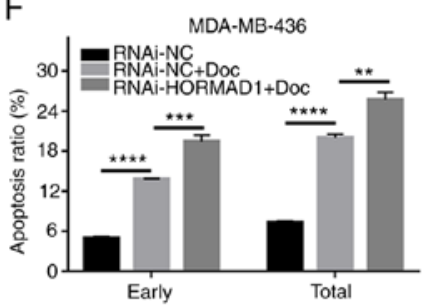

G

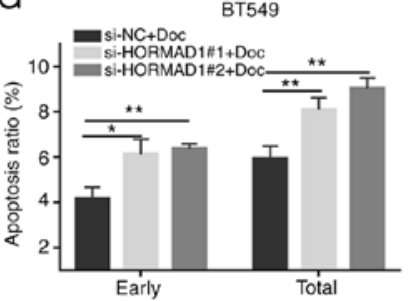

$\mathrm{H}$
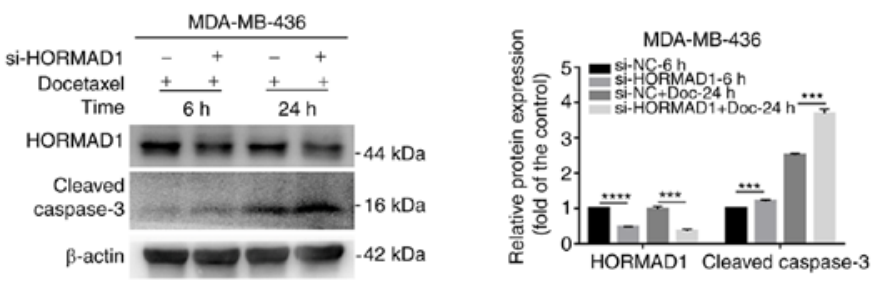

I
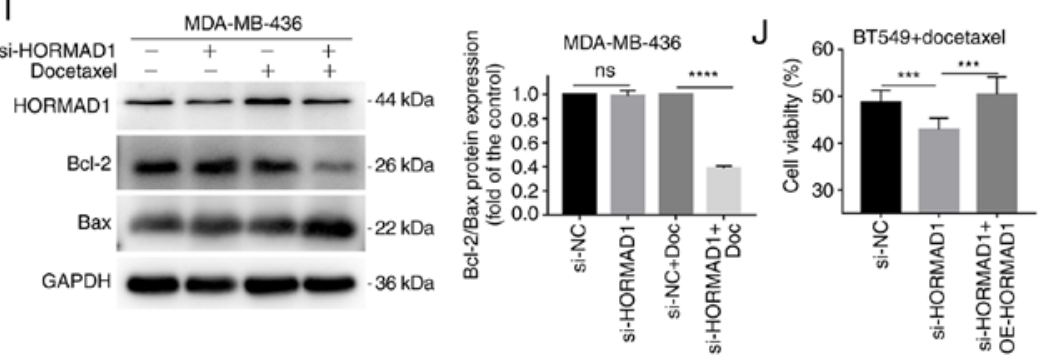

Figure 3. Effects of HORMAD1 knockdown on triple-negative breast cancer cell apoptosis. (A) Cell viability following treatment with different concentrations of Doc for $24 \mathrm{~h}$ in cells with HORMAD1 knockdown was lower than that in control group cells. (B) Similar trends were observed in BT549 cells using two different specific siRNAs. (C) A lentivirus was used as vector to knockdown HORMAD1 expression. The cell growth rate of the RNAi-HORMAD1 group was lower than that of the control group. (D) Similar trends were observed in BT549 cells using two different specific siRNAs. (E and F) Representative images of apoptosis in each group exposed to different treatment concentrations. Doc-induced apoptosis was higher in the HORMAD1 knockdown group compared with the control group. (G) Similar trends were observed in BT549 cells using two different specific siRNAs. (H) Compared with the si-NC group, cleaved caspase-3 expression was significantly increased in the HORMAD1 knockdown group exposed to Doc from 6 to 24 h. (I) HORMAD1 knockdown resulted in a lower Bcl-2/Bax expression upon exposure of MDA-MB-436 cells to Doc for $24 \mathrm{~h}$. (J) The concurrent overexpression of HORMAD1 plasmid-rescued cell survival, compared with the HORMAD1-knockdown group upon exposure of BT549 cells to Doc for $48 \mathrm{~h} .{ }^{* *} \mathrm{P}<0.01,{ }^{* * *} \mathrm{P}<0.005$ and ${ }^{* * * * *} \mathrm{P}<0.001$; HORMAD1, HORMA domain-containing protein 1; Doc, docetaxel; siRNA, small interfering RNA; RNAi-HORMAD1; RNA interfering-HORMAD1; si-NC, siRNA-negative control; si-HORMAD1, siRNA-HORMAD1; ns, no significance. 
A

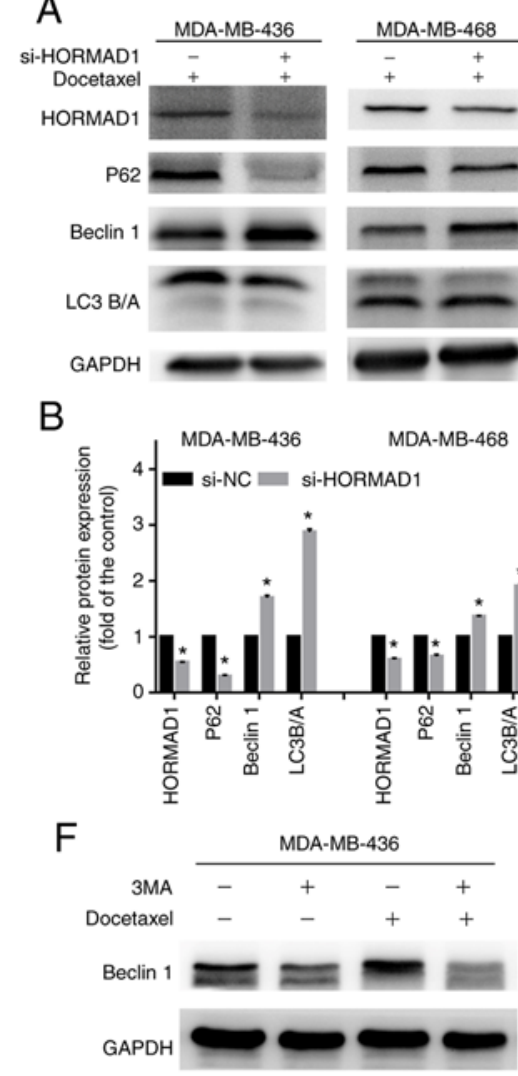

G

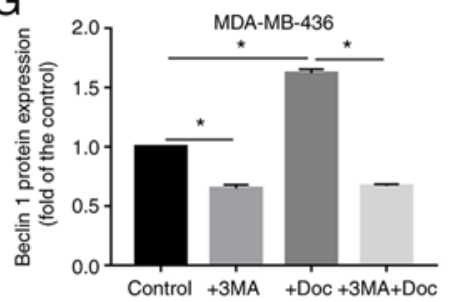

C

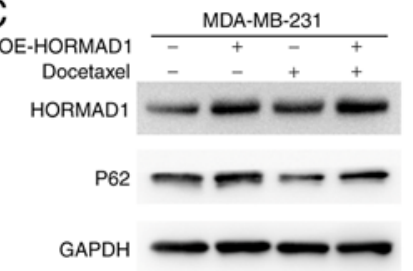

D

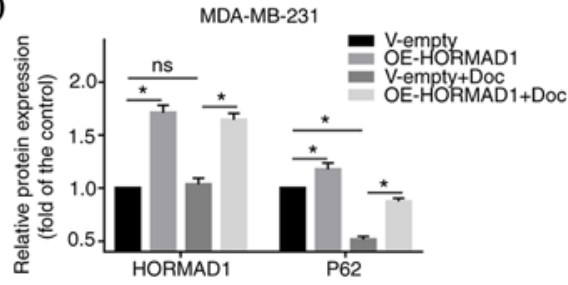

$\mathrm{E}$

MDA-MB-231
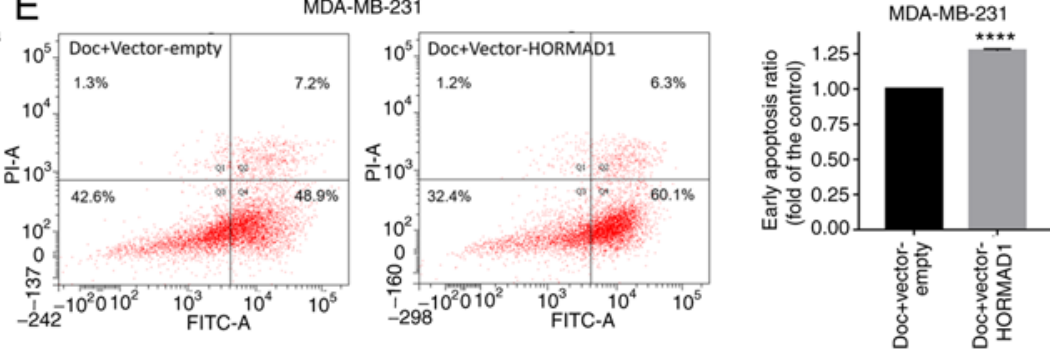

$\mathrm{H}$

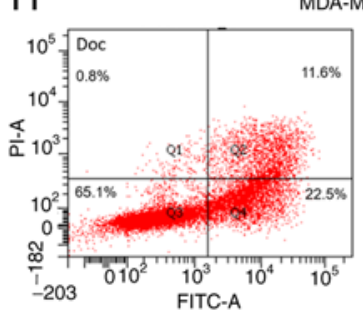

MDA-MB-436
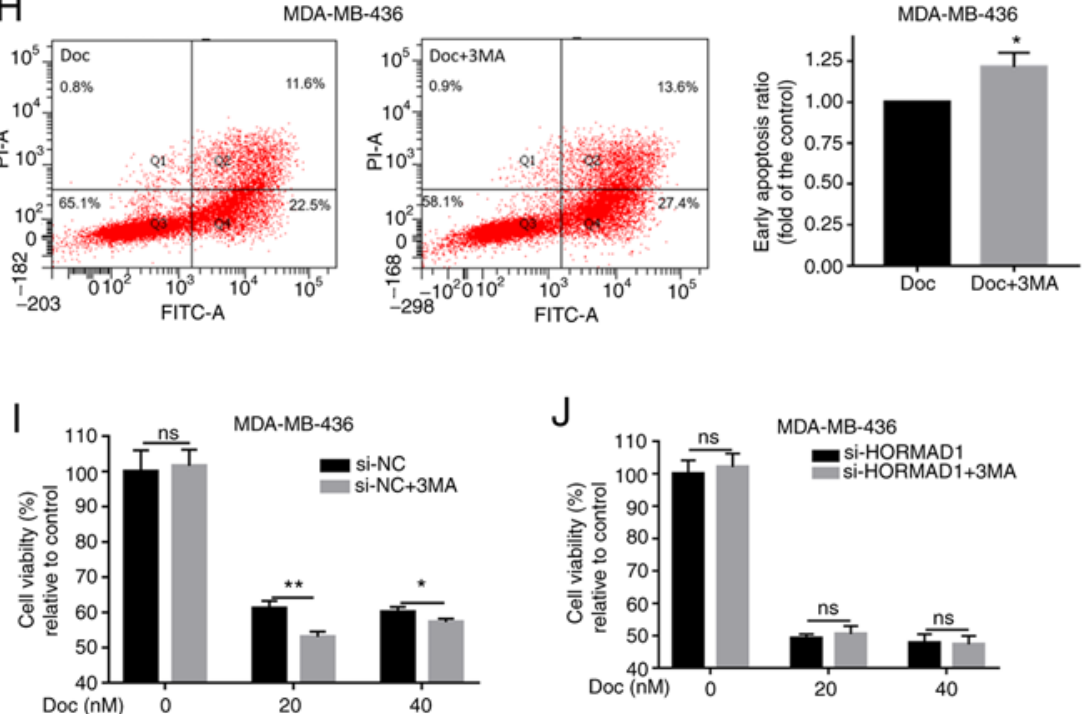

J

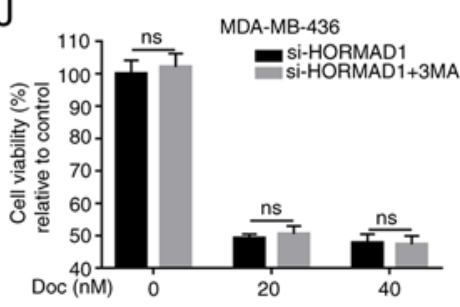

Figure 4. HORMAD1 promotes Doc resistance by not enhancing autophagy. (A and B) Western blot analysis of autophagy markers in MDA-MB-436 and MDA-MB-468 cells. P62 and LC3A were decreased and Beclin 1 was increased in the HORMAD1-knockdown group. (C and D) P62 expression was significantly increased in response to HORMAD1 overexpression in MDA-MB-231 cells. (E) Doc-induced apoptosis for 24 h in MDA-MB-231 cells overexpressing HORMAD1 was higher than that in the control group. (F and G) The addition of 3MA significantly inhibited the expression of Beclin 1, while the addition of Doc significantly increased the expression of Beclin 1. (H) Apoptotic rates induced by Doc for $24 \mathrm{~h}$ in MDA-MB-436 cells treated with $3 \mathrm{MA}$ were higher than those in the control group. (I) The viability of MDA-MB-436 cells treated with 3MA was lower than that of cells in the control group following exposure to Doc for $24 \mathrm{~h}$. (J) Cell viability of MDA-MB-436 treated with si-HORMAD1 was not altered by treatment with Doc for $24 \mathrm{~h}$, with or without 3 MA. GAPDH was used as an internal reference protein. ${ }^{*} \mathrm{P}<0.05,{ }^{* *} \mathrm{P}<0.01$ and ${ }^{* * * *} \mathrm{P}<0.001$. HORMAD1, HORMA domain-containing protein 1; Doc, docetaxel; 3MA, 3-methyladenine; si-NC, small interfering RNA-negative control; si-HORMAD1, siRNA-HORMAD1; ns, no significance; oe, overexpression.

which may be a major contributor to the induction of chemotherapy resistance.

Caspase activity analysis demonstrated that, compared with controls, HORMAD1 knockdown could significantly increase the levels of active caspase-3 (1 vs. 1.63 \pm 0.03 ; $\mathrm{P}<0.001)$, caspase- 8 ( 1 vs. $1.29 \pm 0.05 ; \mathrm{P}<0.01)$ and caspase-9 (1 vs. $1.73 \pm 0.061 ; \mathrm{P}<0.005)$, following exposure to Doc (Fig. 5B). These findings could also explain the increased apoptosis observed in the knockdown group and suggest that the knockdown of HORMAD1 can promote both extrinsic and intrinsic pathways induced by Doc to increase apoptosis.

The detection of DNA damage markers revealed that $\gamma \mathrm{H} 2 \mathrm{AX}$ expression was significantly higher in the HORMAD1 knockdown group $(\mathrm{P}<0.05)$, while that of the DNA repair protein $\mathrm{RAD} 51$ was lower $(\mathrm{P}<0.05)$ than that in the control in MDA-MB-436 cells (Figs. 5C and S2D), MDA-MB-468 (Fig. S2E) and BT549 (Fig. 5D) cells, indicating that there was a significant increase in double-strand breaks in the knockdown group and that the Doc-induced damage tolerance was reduced. The potential role of HORMAD1 in improving DNA damage tolerance has previously been studied in lung cancer (24); in the present study, it was indicated that HORMAD1 plays a similar role in TNBC.

The main changes of apoptosis are the progressive degradation of DNA and the formation of apoptotic bodies. Hoechst staining revealed that cells in different treatment groups demonstrated different proportions of chromatin clumps and nucleate fragmentation. Compared with the control group, cells with Doc treatment exhibited significant nuclear fragmentation. Compared with the si-NC group, the HORMAD1 
A
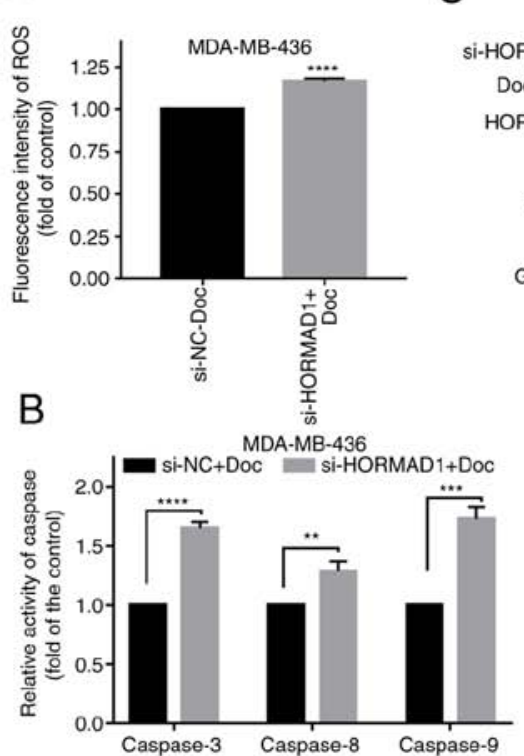

C

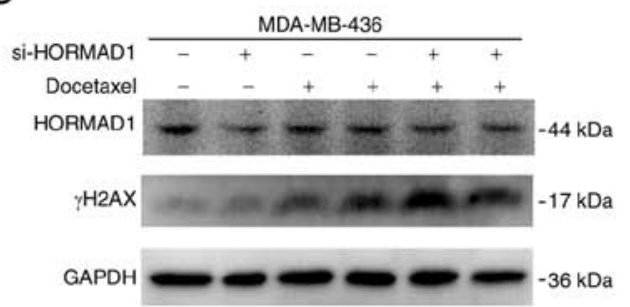

$\mathrm{D}$

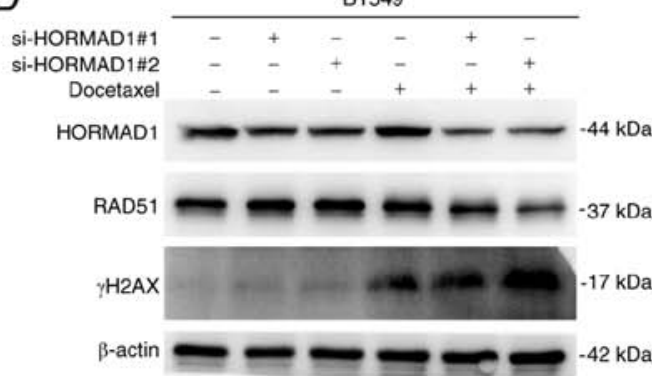

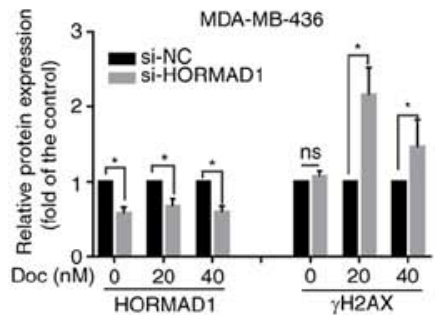

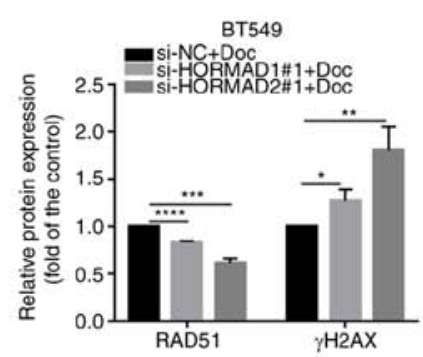

$\mathrm{E}$
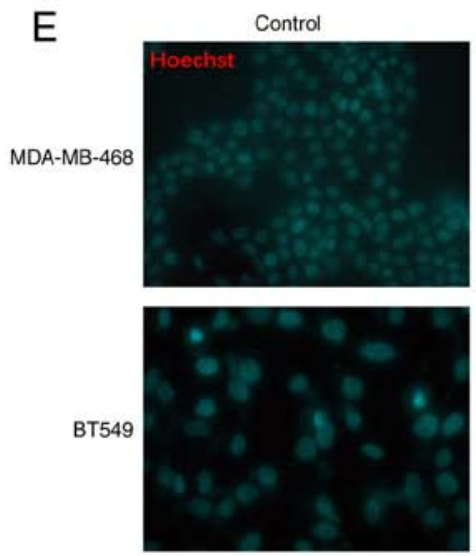
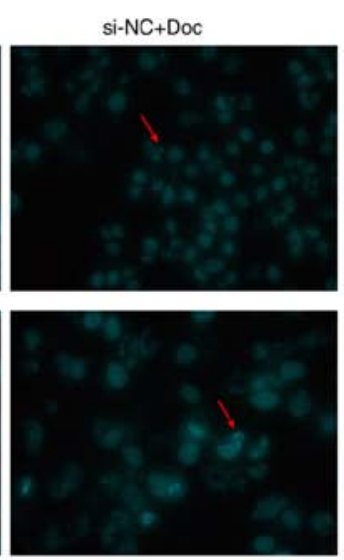

Si-HORMAD1+Doc
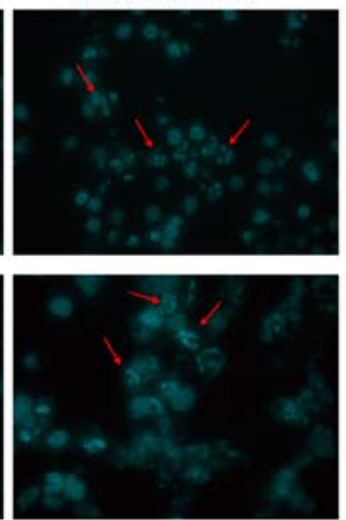

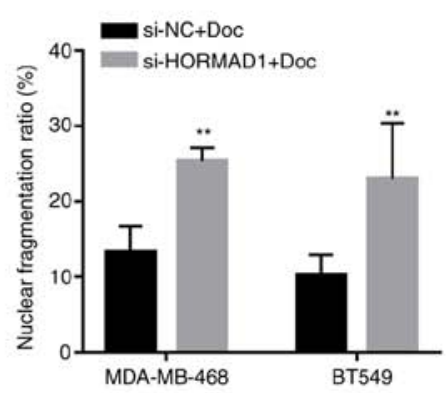

Figure 5. HORMAD1 promotes Doc resistance by enhancing DNA damage tolerance. (A) The induction of intracellular ROS generation was increased by Doc in MDA-MB-436 cells treated with si-HORMAD1 compared with the control group. (B) Caspase enzyme activity in MDA-MB-436 cells treated with si-NC/HORMAD1 following exposure to $40 \mathrm{nM}$ Doc for $24 \mathrm{~h}$. The activity of caspases-3, -8 and -9 was higher than that in the control group. (C) Western blot analysis of DNA damage and repair markers in MDA-MB-436 cells. The $\gamma \mathrm{H} 2 \mathrm{AX}$ expression in the HORMAD1-knockdown group was significantly higher not only in the $20 \mathrm{nM}$ group (lane 3 vs. lane 5) but also in the $40 \mathrm{nM}$ group (lane 4 vs. lane 6) than that induced by Doc (40 nM) in the control group. (D) Western blot analysis of DNA damage and repair protein markers in BT549 cells. The result trend was consistent with that in MDA-MB-436 cells. GAPDH was used as an internal reference protein. (E) Hoechst staining revealed that, compared with the si-NC group, the HORMAD1 knockdown group exhibited a higher proportion of nuclear fragmentation (magnification, $\mathrm{x} 200$; red arrow). ${ }^{*} \mathrm{P}<0.05,{ }^{* * *} \mathrm{P}<0.01,{ }^{* * *} \mathrm{P}<0.005$ and ${ }^{* * * * *} \mathrm{P}<0.001$. HORMAD1, HORMA domain-containing protein 1 ; ROS, reactive oxygen species; Doc, docetaxel; si-HORMAD1, small interfering RNA-HORMAD1; si-NC, siRNA-negative control; ns, no significance.

knockdown group showed a higher proportion of nuclear fragmentation $(\mathrm{P}<0.01$; Fig. 5E).

Apoptosis is closely associated with autophagy (27), and there have been several studies evaluating the correlation between autophagy and drug resistance. These results indicated that HORMAD1 depletion promotes Doc susceptibility caused by a DNA repair defect, which is attributable to a lack of contribution from HORMAD1 to homologous recombination (HR) during TNBC cell mitosis (16).

\section{Discussion}

This study innovatively examined the histological expression of HORMAD1 in TNBC samples with residual lesions after NAC. Concurrently, the present study demonstrated that HORMAD1 and autophagy substrate P62 may be closely related. The role of HORMAD1 in DNA damage tolerance of tumor cells may be the cause of Doc resistance. In the present study, it was demonstrated that HORMAD1 was preferentially overexpressed in the BLBC breast cancer subtype, based on the analysis of TCGA datasets, and that the aberrant expression of HORMAD1 was likely to have a specific biological significance in TNBC. In previous studies, the detection of high HORMAD1 expression levels has generally been conducted at the gene level, with protein levels rarely reported. The proportion of clinical and database samples with high HORMAD1 mRNA expression levels was 52.2-83.6\% (17), while the protein levels of HORMAD1 only detected in TNBC samples were only $29.7 \%$ (22). This difference between gene and protein levels may be due to post-transcriptional translation and modification (28), or the rapid degradation of the protein. In the present study, the analysis of clinical samples 
also revealed that HORMAD1 protein expression was $23.9 \%$ in TNBC tumors from patients treated with NAC. Given the differences in detection reagents and sample processing, this result could reflect the expression of HORMAD1 in TNBC samples; however, HORMAD1 was not found to be a prognostic indicator for TNBC. Based on the results of the bioinformatics analysis of paired tumor and peritumoral samples, and the findings of the laboratory experiments, the present study concluded that HORMAD1 is unlikely to be an independent predictor of prognosis in patients with TNBC, which was inconsistent with the results reported by Chen et al (22), who reported that HORMAD1 is a key differential gene associated with poor outcome in TNBC. This difference may also be due to the fact that the present study followed different types of TNBC patients with or without NAC treatment. Further studies with larger sample sizes and a longer follow-up periods are required to confirm the conclusion of the present study.

HORMAD1 is a specific germ cell protein known to be reactivated in certain cancer types (11). HORMAD1 belongs to a family of proteins [meiosis-specific protein HOP1, mitotic arrest deficient 2 like 2, mitotic arrest deficient 2 like $1(\operatorname{Mad} 2)]$ characterized by a HORMA domain that is present in several DNA repair pathways (29). The best characterized HORMA domain protein, Mad2, is an essential spindle assembly checkpoint mediator. A previous study of HORMAD1 in mice has revealed that it plays an important role in the early detection of meiosis by ensuring the successful search for homologous DNA double-strand breaks and the rapid dissociation and degradation following the formation of normal synaptic complexes (30). HORMAD1 has been proposed to play roles in genomic instability and drug resistance (31) and encodes a HORMAD that binds to DNA double-strand breaks during meiosis, promotes synapsis formation and activates HR (32). In the present experiments, it was demonstrated that cells with HORMAD1 knockdown had a higher apoptotic rate when exposed to Doc treatment; however, the proliferation, cycle and apoptosis of TNBC was not affected by HORMAD1 knockdown with siRNA alone. It was indicated that the role of HORMAD1 in DNA fragmentation and recombination may be the main reason for its role in chemotherapeutic drug resistance.

Autophagy is an evolutionarily conserved, lysosomal dependent, self-degradation pathway that not only plays an important role in maintaining genomic integrity and homeostasis but is also closely associated with tumor development and drug resistance (26). Protective autophagy can promote the survival of drug-resistant cells; however, as a mechanism of programmed cell death, autophagy can directly promote the death of drug-resistant tumor cells. Autophagy can be induced by several factors, such as starvation, growth factor deficiency, microbial infection, organelle damage, protein misfolding or aggregation, DNA damage, radiation and chemotherapy (26). It was confirmed that the transient knockdown of HORMAD1 expression in MDA-MB-436 and MDA-MB-468 cells resulted in changes in autophagy markers, but not in BT 549 cells. In addition, compared with autophagy induced by EBSS-induced, the autophagy associated with HORMAD1 knockdown may be more complex. A previous study reported that autophagy-related protein (Atg) 101, which is a HORMAD, could interact with and stabilize the N-terminal
HORMA domain of Atg13; meanwhile, Atg101 is capable of interacting with the C-terminal domain of Atg1, suggesting that this unique subunit might have functions beyond that of stabilizing Atg13 (33). Future studies should focus on whether HORMAD1, which also contains the HORMA domain, is linked to autophagy.

Treatment with Doc was also accompanied by the activation of autophagy. P62 has been established to contribute to both autophagy and apoptosis in tumor cells. The ubiquitin-associated (UBA) domain of P62 can recruit ubiquitinated proteins, particularly those exposed to oxidants and proteasome inhibitors (34). Therefore, it was hypothesized that autophagy may play a role in Doc resistance. The results revealed that 3MA increased apoptosis in Doc-induced MDA-MB-231 cells with a markedly low HORMAD1 expression, indicating that the inhibition of autophagy could increase drug sensitivity. Concurrently, in MDA-MB-436 cells with a high HORMAD1 expression, 3MA increased the Doc-induced cell viability decrease at low concentrations of Doc $(20 \mathrm{nM})$, indicating that the inhibition of autophagy could increase drug sensitivity. However, with the increase of Doc $(40 \mathrm{nM})$ concentration, this sensitization effect decreased, and the high expression of HORMAD1 was considered to have played an unknown role in it. Drug sensitivity was further detected following HORMAD1 knockdown. The results indicated that HORMAD1 knockdown and the inhibition of autophagy by $3 \mathrm{MA}$ in MDA-MB-436 cells did not further increase sensitivity to Doc.

Cells in the HORMAD1-knockout group produced more ROS following the addition of Doc, which may have been the cause of the increased autophagy in that group. In the present study, the expression of P62 was both increased by the autophagy inhibitor 3MA and induced by HORMAD1 overexpression. Furthermore, both 3MA and HORMAD1 overexpression enhanced the Doc-induced reduction of cell proliferation. In a previous study of glioma (27), it was revealed that caspase- 8 could be activated by wild-type P62 overexpression and then promote HAMLET-induced apoptosis, whereas P62 knockdown had the opposite effect. Furthermore, it was demonstrated that P62 function following HAMLET treatment requires its C-terminus UBA domain. The aforementioned study indicated that, in addition to being a marker of autophagy activation in HAMLET-treated glioma cells, P62 could also function as an important mediator of the activation of caspase-8-dependent cell death (27). In the present study, HORMAD1 knockdown alone could reduce P62 levels and activate autophagy, while HORMAD1 overexpression increased P62 expression; however, the present results also demonstrated that HORMAD1 functioned as an antioxidant and was involved in DNA damage tolerance in TNBC, which potentially plays critical roles in Doc resistance, rather than in its association with autophagy. Whether P62 is important in this process will be addressed in future research.

These results suggested that autophagy may not be the main factor affecting the sensitivity to Doc, but an accompanying effect of the transient knockdown of HORMAD1, which indicates the need for further investigations into the underlying mechanisms. Therefore, the reason why HORMAD1 plays a role in Doc resistance in tumors through its basic physiological functions was explored $(32,35)$. 
Chemotherapy is an important TNBC treatment approach, and taxane chemotherapy is considered one of the most effective strategies (4). Doc is a semi-synthetic derivative of paclitaxel, a widely used chemotherapeutic agent in a variety of cancers. Nevertheless, drug resistance is an urgent problem in the current treatment of tumors, particularly for patients with TNBC. Several studies have investigated the signaling pathways involved in sensitized Doc. Baicalin induced apoptosis inhibited metastasis and enhanced Doc sensitivity through the $\mathrm{NF}-\kappa \mathrm{B}$ signaling pathway in breast cancer cells (36). Another study investigating the effects of HORMAD1 silencing on the gene expression profile of ovarian cancer cells also indicated that the NF- $\kappa \mathrm{B}$ pathway was inhibited (15). The PI3K/AKT $(37,38)$ and JNK pathways (39) have also been revealed to be involved. The study of these involved pathways may contribute to the treatment of cancer.

There are several reasons for Doc resistance, one of which is the abnormal expression of apoptosis-related genes. BRCA1 DNA repair associated (BRCA1) and BRCA2 play an important role in cell proliferation, apoptosis and DNA damage repair. The BRCA1 mutation rate in $\mathrm{TNBC}$ is as high as $71 \%$, and the efficacy of Doc combined with doxorubicin for NAC is poor in patients with breast cancer with germline BRCA1 mutations (40). MDA-MB-436 is a BRCA1 mutant TNBC cell line (41). Cancer cells are highly heterogeneous and cancer cell subtypes can result from the same mutations, while further mutations lead to different subpopulations, which may be partly due to individual decisions made by cells about which survival mechanisms to employ, including HR repair, fork protection or both (42). In BRCA1 mutant tumors, the recovery of HR repair may promote tumor cell survival (43). In a study of TNBC, HORMAD1 was revealed to drive 53BP-1-dependence of non-homologous end-joining (NHEJ) DNA repair (16), while an investigation of non-small cell lung cancer demonstrated that the high HORMAD1 expression led to the expression of a set of HR-related genes, contributing to the formation of RAD51 filaments (24), which also explains the reduced RAD51 expression on HORMAD1 knockdown with Doc treatment, indicating impaired DNA repair ability. A recent study reported that HORMAD1 compromises DNA mismatch repair in cancer cells. It was demonstrated by mass spectrometry that HORMAD1 interacted with MCM8-MCM9 complex and prevented its efficient nuclear localization. As a consequence, HORMAD1-expressing cancer cells exhibited reduced mutL homolog 1 chromatin binding and DNA mismatch repair defects. Therefore, HORMAD1 expression was associated with an increased mutation load and genomic instability in several types of cancer (11). However, whether this is related to the increased Doc sensitivity caused by HORMAD1 knockdown still requires further study. Therefore, it was indicated that, in certain types of TNBC tumors, overexpressing HORMAD1 can repair damaged DNA reduced by Doc, not only through NHEJ but also through HR.

The various mechanisms of cancer resistance that have been identified suggested that tumor cells respond uniquely to individual defects in molecular function rather than to overall genetic defects, to rebalance the intracellular environment and ensure tumor cell survival (43). HORMAD1 reactivation may be one of the manifestations of this mechanism. Several studies have examined the association between HORMAD1 expression and PARPi resistance $(16,17,44)$. It was hypothesized that conventional chemotherapy combined with PARPi or sequential PARPi could be selected for tumors expressing high levels of HORMAD1 (45). The inhibition of PARP expression in cancer tissues may result in minimal side effects from tumor-specific treatment. Given the rapid development of siRNA technology (43), determining the role of HORMAD1 in TNBC, and particularly its mechanism of action in tumor resistance, could enable the relatively specific targeting of HORMAD1 transcripts in TNBC, providing reliable sensitization to treatment. Therefore, the limitation of the present study was that no animal experiments were conducted to verify the results of the cell experiments.

In conclusion, the level of HORMAD1 was revealed to be increased in TNBC tissues and cells. HORMAD1 silencing did not affect TNBC cell proliferation and apoptosis; however, it increased the sensitivity of TNBC cells to Doc. Human TNBC cells were treated with Doc, which led to a significantly decreased cell viability, with an accompanying activation of autophagy; however, the regulatory association between HORMAD1 and autophagy is not the cause of drug resistance; rather the role of HORMAD1 in HR and DNA repair may be the main factor leading to its effects on Doc sensitivity. The present results provided the basis for future studies on the biological functions of HORMAD1 in tumors, particularly TNBC, and for the development of additional therapeutic clinic approaches. Although further biochemical studies are required to characterize the physiological properties of HORMAD1 as an important player in Doc-resistant TNBC cells, the current findings suggested that HORMAD1 may be an attractive target for chemotherapy-mediated TNBC therapy.

\section{Acknowledgements}

We would like to thank the pathologists Dr Hua Liu and Dr Xiao Yang at the Department of Pathology (First Affiliated Hospital of Chongqing Medical University, Chongqing, China) for their help in finding and sectioning tissues of TNBC samples. We would also like to thank the technicians at the laboratory of Chongqing City Key Lab of Translational Medical Research in Cognitive Development and Disorders (Chongqing, China) for the experimental guidance and help.

\section{Funding}

The present study was supported by the National Natural Science Foundation of China (grant no. 81772979).

\section{Availability of data and materials}

All datasets generated during the present study are available from the corresponding author upon reasonable request.

\section{Authors' contributions}

BZ and SL designed the research study. BZ, YP, YW, YZ and YY performed the experiments. BZ, JL, LS, SG and KL analyzed the data. BZ and SL wrote the paper. All authors read and approved the final manuscript. 


\section{Ethics approval and consent to participate}

The present study was approved by the Ethics Committee of the First Affiliated Hospital of Chongqing Medical University (Chongqing, China). The approval number is 2020-279.

\section{Patient consent for publication}

Not applicable.

\section{Competing interests}

The authors declare that they have no competing interests.

\section{References}

1. Erratum: Global cancer statistics 2018: GLOBOCAN estimates of incidence and mortality worldwide for 36 cancers in 185 countries. CA Cancer J Clin 70: 313, 2020.

2. den Brok WD, Speers CH, Gondara L, Baxter E, Tyldesley SK and Lohrisch CA: Survival with metastatic breast cancer based on initial presentation, de novo versus relapsed. Breast Cancer Res Treat 161: 549-556, 2017.

3. Ruddy KJ and Ganz PA: Treatment of nonmetastatic breast cancer. JAMA 321: 1716-1717, 2019.

4. Gradishar WJ, Anderson BO, Abraham J, Aft R, Agnese D, Allison KH, Blair SL, Burstein HJ, Dang C, Elias AD, et al: Breast cancer, version 3.2020, NCCN clinical practice guidelines in oncology. J Natl Compr Canc Netw 18: 452-478, 2020.

5. Cardoso F, Senkus E, Costa A, Papadopoulos E, Aapro M, André F, Harbeck N, Aguilar Lopez B, Barrios CH, Bergh J, et al: 4th ESO-ESMO international consensus guidelines for advanced breast cancer (ABC 4)†. Ann Oncol 29: 1634-1657, 2018.

6. National Comprehensive Cancer Network: NCCN Clinical Practice Guidelines in Oncology-Breast Cancer (Version 1, 2018), [DB/OL]. http://www.nccn.org.

7. Liu P, Kumar IS, Brown S, Kannappan V, Tawari PE, Tang JZ, Jiang W, Armesilla AL, Darling JL and Wang W: Disulfiram targets cancer stem-like cells and reverses resistance and cross-resistance in acquired paclitaxel-resistant triple-negative breast cancer cells. Br J Cancer 109: 1876-1885, 2013.

8. Byrski T, Gronwald J, Huzarski T, Grzybowska E, Budryk M, Stawicka M, Mierzwa T, Szwiec M, Wisniowski R, Siolek M, et al: Pathologic complete response rates in young women with BRCA1-positive breast cancers after neoadjuvant chemotherapy. J Clin Oncol 28: 375-379, 2010.

9. Silver DP, Richardson AL, Eklund AC, Wang ZC, Szallasi Z, Li Q, Juul N, Leong CO, Calogrias D, Buraimoh A, et al: Efficacy of neoadjuvant cisplatin in triple-negative breast cancer. J Clin Oncol 28: 1145-1153, 2010.

10. Asano Y, Kashiwagi S, Goto W, Takada K, Takahashi K, Morisaki T, Fujita H, Takashima T, Tomita S, Ohsawa M, et al: Prediction of treatment responses to neoadjuvant chemotherapy in triple-negative breast cancer by analysis of immune checkpoint protein expression. J Transl Med 16: 87, 2018.

11. Liu K, Wang Y, Zhu Q, Li P, Chen J, Tang Z, Shen Y, Cheng X, Lu LY and Liu Y: Aberrantly expressed HORMAD1 disrupts nuclear localization of MCM8-MCM9 complex and compromises DNA mismatch repair in cancer cells. Cell Death Dis 11: 519,2020

12. Qiao H, Rao HBDP, Yun Y, Sandhu S, Fong JH, Sapre M, Nguyen M, Tham A, Van BW, Chng TYH, et al: Impeding DNA break repair enables oocyte quality control. Mol Cell 72: 211-221.e3, 2018.

13. Chen YT, Venditti CA, Theiler G, Stevenson BJ, Iseli C, Gure AO, Jongeneel CV, Old LJ and Simpson AJ: Identification of CT46/HORMAD1, an immunogenic cancer/testis antigen encoding a putative meiosis-related protein. Cancer Immun 5: 9, 2005.

14. Adélaïde J, Finetti P, Bekhouche I, Repellini L, Geneix J, Sircoulomb F, Charafe-Jauffret E, Cervera N, Desplans J, Parzy D, et al: Integrated profiling of basal and luminal breast cancers. Cancer Res 67: 11565-11575, 2007.

15. Shahzad MM, Shin YH, Matsuo K, Lu C, Nishimura M, Shen DY, Kang Y, Hu W, Mora EM, Rodriguez-Aguayo C, et al: Biological significance of HORMA domain containing protein 1 (HORMAD1) in epithelial ovarian carcinoma. Cancer Lett 330 : 123-129, 2013
16. Watkins J, Weekes D, Shah V, Gazinska P, Joshi S, Sidhu B, Gillett C, Pinder S, Vanoli F, Jasin M, et al: Genomic complexity profiling reveals that HORMAD1 overexpression contributes to homologous recombination deficiency in triple-negative breast cancers. Cancer Discov 5: 488-505, 2015.

17. Wang X, Tan Y, Cao X, Kim JA, Chen T, Hu Y, Wexler M and Wang X: Epigenetic activation of HORMAD1 in basal-like breast cancer: Role in Rucaparib sensitivity. Oncotarget 9: 30115-30127, 2018.

18. Guo SP,Jian L, Tao K, Chen C, Yu H and Liu S: Novel breast-specific long non-coding RNA LINC00993 acts as a tumor suppressor in triple-negative breast cancer. Front Oncol 9: 1325, 2019.

19. Gebhart G, Lamberts L, Wimana Z, Garcia C, Emonts P, Ameye L, Stroobants S, Huizing M, Aftimos P, Tol J, et al: Molecular imaging as a tool to investigate heterogeneity of advanced HER2-positive breast cancer and to predict patient outcome under trastuzumab emtansine (T-DM1): The ZEPHIR trial. Ann Oncol 27: 619-624, 2016.

20. Livak KJ and Schmittgen TD: Analysis of relative gene expression data using real-time quantitative PCR and the 2(-Delta Delta C(T)) method. Methods 25: 402-408, 2001.

21. Basu A: Regulation of autophagy by protein kinase C- $\varepsilon$ in breast cancer cells. Int J Mol Sci 21: 4247, 2020.

22. Chen B, Tang H, Chen X, Zhang G, Wang Y, Xie X and Liao N: Transcriptomic analyses identify key differentially expressed genes and clinical outcomes between triple-negative and non-triple-negative breast cancer. Cancer Manag Res 11: 179-190, 2018.

23. Tomioka N, Azuma M, Ikarashi M, Yamamoto M, Sato M, Watanabe KI, Yamashiro K and Takahashi M: The therapeutic candidate for immune checkpoint inhibitors elucidated by the status of tumor-infiltrating lymphocytes (TILs) and programmed death ligand 1 (PD-L1) expression in triple negative breast cancer (TNBC). Breast Cancer 25: 34-42, 2018.

24. Nichols BA, Oswald NW, McMillan EA, McGlynn K, Yan J, Kim MS, Saha J, Mallipeddi PL, LaDuke SA, Villalobos PA, et al: HORMAD1 is a negative prognostic indicator in lung adenocarcinoma and specifies resistance to oxidative and genotoxic stress. Cancer Res 78: 6196-6208, 2018.

25. Yao J, Caballero OL, Yung WK, Weinstein JN, Riggins GJ, Strausberg RL and Zhao Q: Tumor subtype-specific cancer-testis antigens as potential biomarkers and immunotherapeutic targets for cancers. Cancer Immunol Res 2: 371-379, 2014.

26. Levine B and Kroemer G: Biological functions of autophagy genes: A disease perspective. Cell 176: 11-42, 2019.

27. Zhang YB, Gong JL, Xing TY, Zheng SP and Ding W: Autophagy protein p62/SQSTM1 is involved in HAMLET-induced cell death by modulating apotosis in U87MG cells. Cell Death Dis 4: e550, 2013.

28. Chick JM, Munger SC, Simecek P, Huttlin EL, Choi K, Gatti DM, Raghupathy N, Svenson KL, Churchill GA and Gygi SP: Defining the consequences of genetic variation on a proteome-wide scale. Nature 534: 500-505, 2016.

29. Aravind L and Koonin E: The HORMA domain: A common structural denominator in mitotic checkpoints, chromosome synapsis and DNA repair. Trends Biochem Sci 23: 284-286, 1998.

30. Daniel K, Lange J, Hached K, Fu J, Anastassiadis K, Roig I, Cooke HJ, Stewart AF, Wassmann K, Jasin M, et al: Meiotic homologue alignment and its quality surveillance are controlled by mouse HORMAD1. Nat Cell Biol 13: 599-610, 2011.

31. Nielsen AY and Gjerstorff MF: Ectopic expression of testis germ cell proteins in cancer and its potential role in genomic instability. Int J Mol Sci 17: 890, 2016.

32. Shin YH, Choi Y, Erdin SU, Yatsenko SA, Kloc M, Yang F, Wang PJ, Meistrich ML and Rajkovic A: Hormad1 mutation disrupts synaptonemal complex formation, recombination, and chromosome segregation in mammalian meiosis. PLoS Genet 6: e1001190, 2010.

33. Nanji T, Liu X, Chew LH, Li FK, Biswas M, Yu ZQ, Lu S, Dong MQ, Du LL, Klionsky DJ and Yip CK: Conserved and unique features of the fission yeast core Atg1 complex. Autophagy 13: 2018-2027, 2017.

34. Galluzzi L, Baehrecke EH, Ballabio A, Boya P, Bravo-San Pedro JM, Cecconi F, Choi AM, Chu CT, Codogno P, Colombo MI, et al: Molecular definitions of autophagy and related processes. EMBO J 36: 1811-1836, 2017.

35. Acquaviva L, Boekhout M, Karasu ME, Brick K, Pratto F, Li T, van Overbeek M, Kauppi L, Camerini-Otero RD, Jasin M and Keeney S: Ensuring meiotic DNA break formation in the mouse pseudoautosomal region. Nature 582: 426-431, 2020. 
36. Zeng A, Liang $X$, Zhu S, Liu C, Luo X, Zhang Q and Song L: Baicalin, a potent inhibitor of NF- $\kappa \mathrm{B}$ signaling pathway, enhances chemosensitivity of breast cancer cells to docetaxel and inhibits tumor growth and metastasis both in vitro and in vivo. Front Pharmacol 11: 879, 2020.

37. Singh SK, Apata T, Gordetsky JB and Singh R: Docetaxe combined with thymoquinone induces apoptosis in prostate cancer cells via inhibition of the PI3K/AKT signaling pathway. Cancers (Basel) 11: 1139, 2019.

38. Zhang X, Shao J, Li X, Cui L and Tan Z: Docetaxel promotes cell apoptosis and decreases SOX2 expression in CD133-expressing hepatocellular carcinoma stem cells by suppressing the PI3K/AKT signaling pathway. Oncol Rep 41: 1067-1074, 2019.

39. Dávila-GonzálezD, ChoiDS, RosatoRR, Granados-Principal SM, Kuhn JG, Li WF, Qian W, Chen W, Kozielski AJ, Wong H, et al: Pharmacological inhibition of NOS activates ASK1/JNK pathway augmenting docetaxel-mediated apoptosis in triple-negative breast cancer. Clin Cancer Res 24: 1152-1162, 2018.

40. Byrski T, Gronwald J, Huzarski T, Grzybowska E, Budryk M, Stawicka M, Mierzwa T, Szwiec M, Wiśniowski R, Siolek M, et al: Response to neo-adjuvant chemotherapy in women with BRCA1-positive breast cancers. Breast Cancer Res Treat 108: 289-296, 2008
41. Du Y, Yamaguchi H, Wei Y, Hsu JL, Wang HL, Hsu YH, Lin WC Yu WH, Leonard PG, Lee GR IV, et al: Blocking c-Met-mediated PARP1 phosphorylation enhances anti-tumor effects of PARP inhibitors. Nat Med 22: 194-201, 2016.

42. Schlacher K: A new road to cancer-drug resistance. Nature 563: 478-480, 2018

43. Shahzad MM, Lu C, Lee JW, Stone RL, Mitra R, Mangala LS, Lu Y, Baggerly KA, Danes CG, Nick AM, et al: Dual targeting of EphA2 and FAK in ovarian carcinoma. Cancer Biol Ther 8: $1027-1034,2009$

44. Gao Y, Kardos J, Yang Y, Tamir TY, Mutter-Rottmayer E, Weissman B, Major MB, Kim WY and Vaziri C: The cancer/testes (CT) antigen HORMAD1 promotes homologous recombinational DNA repair and radioresistance in lung adenocarcinoma cells. Sci Rep 8: 15304, 2018.

45. Dubois C, Martin F, Hassel C, Magnier F, Daumar P, Aubel C, Guerder S, Mounetou E, Penault-Lorca F and Bamdad M: Low-dose and long-term Olaparib treatment sensitizes MDA-MB-231 and SUM1315 triple-negative breast cancers spheroids to fractioned radiotherapy. J Clin Med 9: 64, 2019.

This work is licensed under a Creative Commons Attribution-NonCommercial-NoDerivatives 4.0 International (CC BY-NC-ND 4.0) License. 